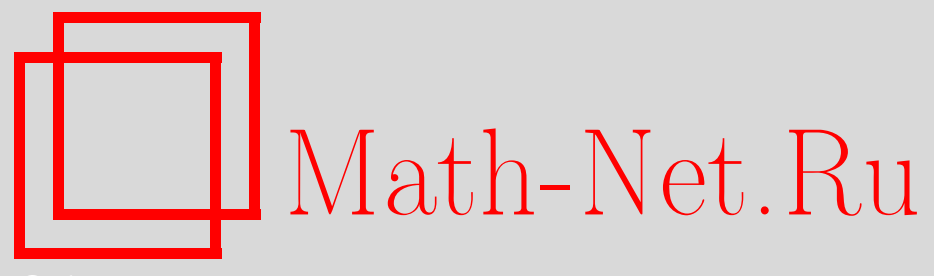

А. В. Разумов, Ю. Г. Строганов, Перечисление матриц чередующихся знаков нечетного порядка, симметричных относительно поворота на $180^{\circ}, T M \Phi, 2006$, том 148 , номер 3, 357-386

DOI: https://doi.org/10.4213/tmf2322

Использование Общероссийского математического портала Math-Net.Ru подразумевает, что вы прочитали и согласны с пользовательским соглашением http://www.mathnet.ru/rus/agreement

Параметры загрузки:

IP : 54.197 .130 .99

26 апреля 2023 г., 12:20:44

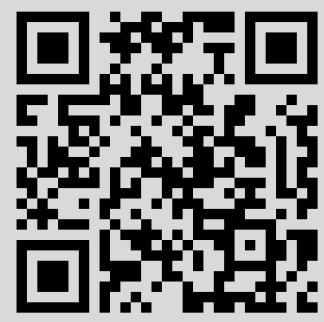




\title{
ПЕРЕЧИСЛЕНИЕ МАТРИЦ ЧЕРЕДУЮЩИХСЯ ЗНАКОВ НЕЧЕТНОГО ПОРЯДКА, СИММЕТРИЧНЫХ ОТНОСИТЕЛЬНО ПОВОРОТА НА $180^{\circ}$
}

\begin{abstract}
Купербергом было показано, что статистическая сумма модели квадратного льда, связанная с матрицами чередующихся знаков четного порядка, симметричными относительно поворота на $180^{\circ}$, является произведением двух схожих множителей. Предлагается модель квадратного льда, состояния которой находятся во взаимно однозначном соответствии с матрицами чередующихся знаков нечетного порядка, симметричными относительно поворота на $180^{\circ}$. Статистическая сумма этой модели выражается через вышеупомянутые множители. Найдены вклады в статистическую сумму, соответствующие матрицам чередующихся знаков, имеющим 1 или -1 в центре матрицы, и найдены соответствующие перечисления.
\end{abstract}

Ключевые слова: матрицы чередующихся знаков, перечисления, модель квадратного льда.

\section{1. ВВЕДЕНИЕ}

Матрица чередующихся знаков - это матрица с элементами 1,0, и -1 такая, что 1 и -1 чередуются в каждом столбце и в каждой строке и, кроме того, первый и последний ненулевые элементы в каждом столбце и в каждой строке равны 1. Начиная с известных гипотез Миллса, Роббинса и Рамсея [1], было сделано много утверждений о перечислениях и о равенствах перечислений, относящихся к матрицам чередующихся знаков и к их разнообразным подклассам. Большинство результатов было доказано путем использования взаимно однозначного соответствия между матрицами и состояниями различных вариантов статистической модели квадратного льда. Впервые этот метод решения задач перечисления был использован Купербергом [2], см. также богатую результатами работу [3].

Настоящая статья посвящена перечислению матриц чередующихся знаков нечетного порядка, симметричных относительно поворота на $180^{\circ}$, на основе соответствующей модели квадратного льда.

* Институт физики высоких энергий, Протвино, Московская обл., Россия. E-mail: Alexander.Razumov@ihep.ru, Yuri.Stroganov@ihep.ru 
В разделе 2 мы напоминаем определение модели квадратного льда с граничными условиями типа доменной стенки. Состояния этой модели находятся во взаимно однозначном соответствии с матрицами чередующихся знаков. Сформулированы необходимые свойства статистической суммы этой модели.

В разделе 3 мы сначала обсуждаем модель квадратного льда, связанную с матрицами чередующихся знаков четного порядка, симметричными относительно поворота на $180^{\circ}$, которая была предложена Купербергом [3]. Затем вводится модель квадратного льда, состояния которой находятся во взаимно однозначном соответствии с матрицами чередующихся знаков нечетного порядка, симметричными относительно поворота на $180^{\circ}$. Мы доказываем, что статистическая сумма этой модели выражается через статистическую сумму модели квадратного льда с граничными условиями типа доменной стенки и статистическую сумму модели, связанную с матрицами чередующихся знаков четного порядка, симметричными относительно поворота на $180^{\circ}$. Оказывается, что можно разделить вклады в статистическую сумму от состояний, соответствующих матрицам чередующихся знаков, имеющим 1 или -1 в центре матрицы (теорема 2).

В разделе 4 мы рассматриваем важный специальный случай, когда общий для всех вершин параметр модели $a=e^{i \pi / 3}$, что позволяет, в частности, доказать перечислительную гипотезу Роббинса [4], относящуюся к матрицам чередующихся знаков нечетного порядка, симметричным относительно поворота на $180^{\circ}$. Интересно, что в этом случае существует факторизованное детерминантное представление для статистической суммы (теорема 3 ).

В разделе 5 мы соотносим перечисления матриц чередующихся знаков нечетного порядка, симметричных относительно поворота на $180^{\circ}$, с перечислениями всех матриц чередующихся знаков и матриц чередующихся знаков четного порядка, симметричных относительно поворота на $180^{\circ}$. В частности, мы находим в явном виде отдельные детальные перечисления матриц чередующихся знаков нечетного порядка, симметричных относительно поворота на $180^{\circ}$, имеющих 1 или -1 в центре матрицы.

\section{2. МОДЕЛЬ КВАДРАТНОГО ЛЬДА С ГРАНИЧНЫМИ УСЛОВИЯМИ ТИПА ДОМЕННОЙ СТЕНКИ}

2.1. Определение модели. Метод, использованный Купербергом для доказательства гипотезы о матрицах чередующихся знаков, основан на взаимно однозначном соответствии между состояниями модели квадратного льда с граничными условиями типа доменной стенки и матрицами чередующихся знаков. Чтобы определить пространство состояний модели квадратного льда, рассмотрим такое подмножество вершин и ребер квадратной решетки, что каждая внутренняя вершина четырехвалентна и каждая граничная вершина одновалентна. Состояние соответствующей модели льда определяется такой ориентацией ребер, что каждая четырехвалентная вершина имеет два входящих ребра и два выходящих. Условия типа доменной стенки [5] фиксируют ориентацию ребер, принадлежащих одновалентным вершинам, в соответствии с образцом, приведенным на рис. 1. Метки $x_{i}$ и $y_{i}$ означают спек- 


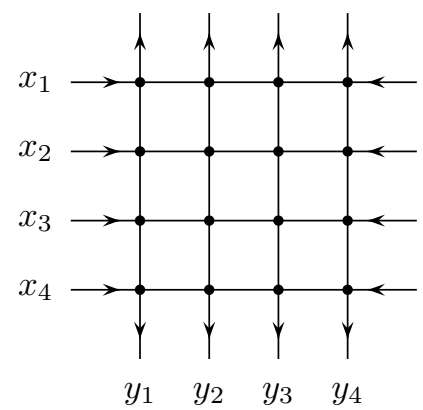

Рис. 1. Квадратный лед с границей типа доменной стенки.

тральные параметры, которые будут использованы для определения статистической суммы модели.

Если каждую четырехвалентную вершину, принадлежащую состоянию модели квадратного льда с граничными условиями типа доменной стенки, заменить числом согласно рис. 2, то мы получим матрицу чередующихся знаков. Нетрудно про-

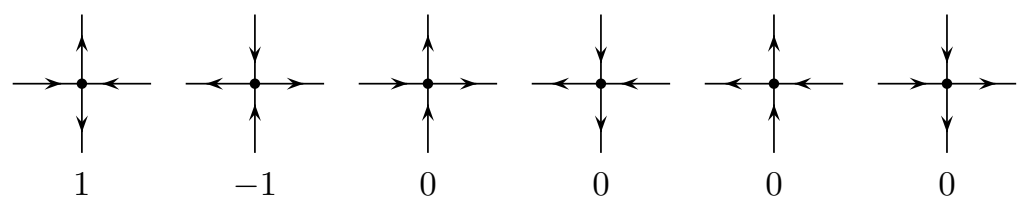

Рис. 2. Соответствие между вершинами квадратного льда и элементами матриц чередующихся знаков.

верить, что таким образом мы получаем взаимно однозначное соответствие между состояниями и матрицами чередующихся знаков [6].

Статистическая сумма квадратного льда с граничными условиями типа доменной стенки является суммой весов всех возможных состояний модели. Вес состояния равен произведению весов всех четырехвалентных вершин. Чтобы определить их, мы связываем спектральные параметры $x_{i}$ с горизонтальными линиями решетки, а спектральные параметры $y_{i}-$ с вертикальными линиями (см. рис. 1). Вершине, находящейся на пересечении линий со спектральными параметрами $x_{i}$ и $y_{j}$, приписывается спектральный параметр $x_{i} \bar{y}_{j}$, где мы используем обозначение $\bar{x}=x^{-1}$, введенное Купербергом. После этого мы определяем веса вершин в соответствии с рис. 3 , где $a$ - общий для всех вершин параметр и использовано удобное обозначение $\sigma(x)=x-\bar{x}$, также введенное Купербергом.

Граф с параметризованными вершинами, подобный тому, который приведен на рис. 1, означает соответствующую статистическую сумму. При этом подразумевается суммирование по всем возможным ориентациям внутренних ребер. Если граничные ребра не имеют ориентации, то граф представляет множество величин, соответствующих всем их возможным ориентациям. Удобно сделать формализм инвариантным по отношению к вращениям. Для этого мы позволяем вершинной метке 


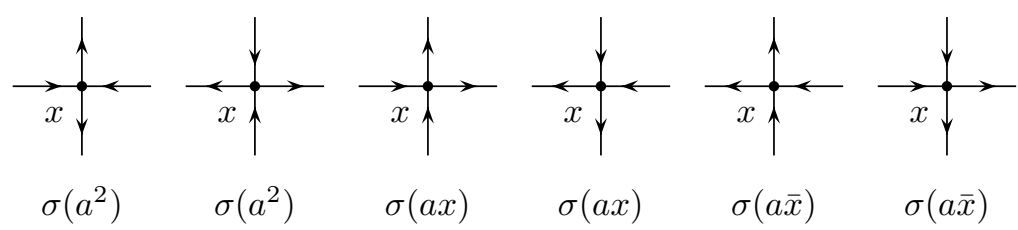

Рис. 3. Веса вершин.

располагаться в любом квадранте, относящемся к вершине. Значение соответствующего спектрального параметра равно $x_{i} \bar{y}_{j}$, если метка размещена в квадранте, заметаемом линией со спектральным параметром $x_{i}$, когда она вращается против часовой стрелки к линии со спектральным параметром $y_{j}$. Из этого правила следует, что мы можем перемещать метку $x$ из одного квадранта в смежный с ним, заменяя ее на $\bar{x}$.

В качестве примера приведем граф, соответствующий хорошо известному уравнению Янга-Бакстера, см. рис. 4. Это уравнение выполняется при условии $x y z=a$. Заметим, что мы используем предложенную Купербергом параметризацию весов,

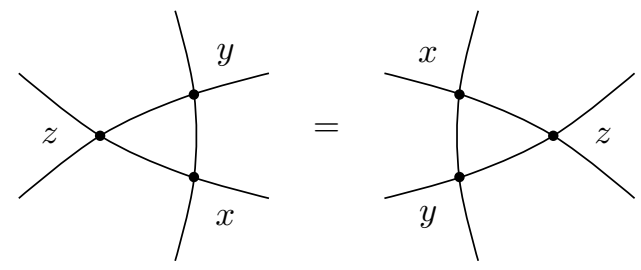

Рис. 4. Уравнение Янга-Бакстера.

удобную для рассмотрения комбинаторных проблем.

2.2. Две леммы о перестановках. Простейшим примером матрицы чередующихся знаков является матрица перестановки, определяемая как матрица, которую можно получить перестановкой строк и столбцов единичной матрицы. Для любой $(n \times n)$-матрицы перестановки $\Sigma$ можно написать

$$
(\Sigma)_{i j}=\delta_{i s(j)}
$$

где $s$ - подходящий единственный элемент симметрической группы $S_{n}$. Очевидно, что если мы идем вдоль столбца $\Sigma$ с номером $j$, то мы встретим 1 в строке с номером $s(j)$, а если мы идем вдоль строки $\Sigma$ с номером $i$, то мы встретим 1 в столбце с номером $s^{-1}(i)$, см. рис. 5 .

Напомним, что пара $(i, j)$ целых чисел таких, что $1 \leqslant i<j \leqslant n$, называется инверсией перестановки $s \in S_{n}$, если $s(i)>s(j)$. Число инверсий $s$ обозначается как $\operatorname{inv}(s)$, мы называем его длиной перестановки. Ясно, что минимальное число 


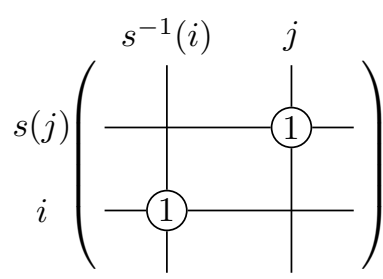

Рис. 5. Позиции единичных элементов в строках и столбцах матрицы перестановки.

инверсий элемента $S_{n}$ равно нулю, а максимальное равно $n(n-1) / 2$. Введем производящую функцию

$$
\Phi(n ; z)=\sum_{s \in S_{n}} z^{\operatorname{inv}(s)}=\sum_{k=0}^{n(n-1) / 2} I(n ; k) z^{k}
$$

где $I(n ; k)$ - число элементов $S_{n}$ с $k$ инверсиями. Хорошо известен следующий результат.

ЛЕмма 1. Для любого положительного иелого $n$ имеем

$$
\Phi(n ; z)=(1+z)\left(1+z+z^{2}\right) \ldots\left(1+z+\cdots+z^{n-1}\right) .
$$

ДокАзАтельство. Сама форма утверждения леммы предлагает доказывать его по индукции. Представим элемент $s$ группы $S_{n}$ как слово $s(1) s(2) \ldots s(n)$. Слова, представляющие элементы $S_{n+1}$, могут быть образованы вставкой буквы $n+1$ в слова, представляющие элементы $S_{n}$. В результате мы имеем $n+1$ возможность вставки. Пусть сначала буква $n+1$ находится в последней позиции получающегося слова. Длина полученного элемента $S_{n+1}$ равна длине $S_{n}$. Такая вставка дает вклад в $\Phi(n+1 ; z)$, совпадающий с $\Phi(n ; z)$. Пусть теперь буква $n+1$ находится в предпоследней позиции. Длина полученной перестановки на единицу больше длины исходной. Это дает вклад $\Phi(n ; z) z$. Исчерпав все возможности, мы получаем равенство

$$
\Phi(n+1 ; z)=\Phi(n ; z)\left(1+z+\cdots+z^{n}\right) .
$$

Принимая во внимание, что $\Phi(1 ; z)=1$, приходим к утверждению леммы.

Лемма 2. Для состояния квадратного льда, соответствующего матрице перестановки, ассоциированной с перестановкой $s \in S_{n}$, числа вериин третвего ти$n a^{1)}$ и четвертого типа равны $\operatorname{inv}(s)$, а числа вериин пятого типа и шестого типа равны $n(n-1) / 2-\operatorname{inv}(s)$.

ДокАЗАтЕЛЬСтво. Рассмотрим состояние квадратного льда, соответствующее матрице перестановки $\Sigma$, ассоциированной с перестановкой $s \in S_{n}$. Заметим, что

\footnotetext{
1) Типы вершин, приведенные на рис. 2, нумеруются слева направо.
} 
если мы двигаемся вдоль строки или столбца на рисунке, представляющем рассматриваемое состояние, то, проходя вершину первого или второго типа, мы меняем ориентацию ребра. Принимая во внимание, что вершины первого или второго типов встречаются там, где мы находим 1 в соответствующей матрице перестановки, приходим к следующему заключению. Ноль в матрице перестановки соответствует вершине третьего типа, если 1 находится ниже его и справа от него; ноль в матрице перестановки соответствует вершине четвертого типа, если 1 находится выше его и слева от него, см. рис. 6. Аналогично, ноль в матрице перестановки соответствует
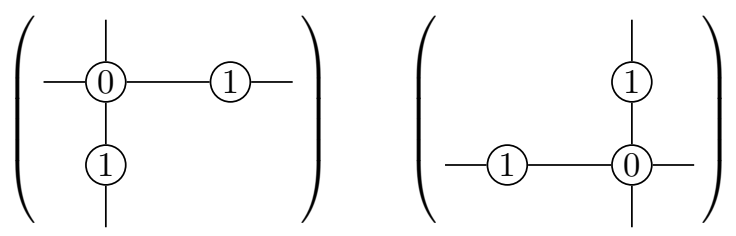

Рис. 6. Расположение вершин третьего и четвертого типов.

вершине пятого типа, если 1 находится ниже его и слева от него; а ноль в матрице перестановки соответствует вершине шестого типа, если 1 находится выше его и справа от него, см. рис. 7.
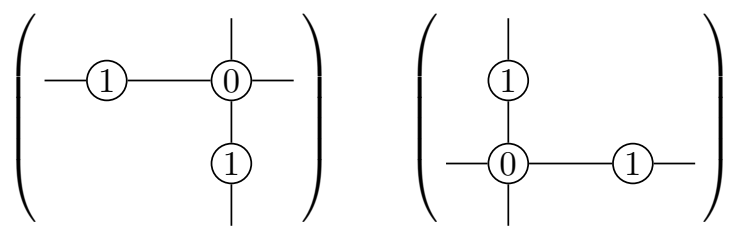

Рис. 7. Расположение вершин пятого и шестого типов.

Пусть теперь $(i, j)$ является инверсией $s$. Рассмотрим элемент, находящийся на пересечении строки с номером $s(i)$ и столбца с номером $j$ в матрице $\Sigma$. Так как $(i, j)$ является инверсией, то мы встречаемся с ситуацией, изображенной на рис. 8. Ясно, что состояние квадратного льда имеет в рассматриваемой позиции вершину

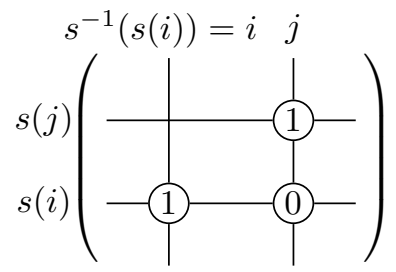

Рис. 8. Соответствие между инверсиями и вершинами четвертого типа.

четвертого типа. Нетрудно убедиться, что мы приходим к взаимно однозначному соответствию между инверсиями $s$ и вершинами четвертого типа. Следовательно, 
число вершин четвертого типа равно $\operatorname{inv}(s)$. Анализируя рис. 6, мы видим, что имеется одинаковое число вершин третьего типа и четвертого типа.

Далее, рис. 7 показывает, что имеется одинаковое число вершин пятого типа и шестого типа. Принимая во внимание, что полное число вершин первого типа и второго типа равно $n$, получаем последнее утверждение леммы.

2.3. Ведущие члены статистической суммы. Обозначим статистическую сумму модели квадратного льда с граничными условиями типа доменной стенки как $Z(n ; \boldsymbol{x}, \boldsymbol{y})$. Здесь $n$ является размером квадратного льда, а $\boldsymbol{x}$ и $\boldsymbol{y}$ - это векторы спектральных параметров:

$$
\boldsymbol{x}=\left(x_{1}, \ldots, x_{n}\right), \quad \boldsymbol{y}=\left(x_{1}, \ldots, x_{n}\right) .
$$

Ясно, что статистическая сумма $Z(n ; \boldsymbol{x}, \boldsymbol{y})$ является полиномом Лорана по переменным $x_{i}$ и $y_{i}$. Определим модифицированную статистическую сумму

$$
\widetilde{Z}(n ; \boldsymbol{x}, \boldsymbol{y})=\left[\prod_{i=1}^{n} x_{i}^{n-1} y_{i}^{n-1}\right] Z(n ; \boldsymbol{x}, \boldsymbol{y}) .
$$

Следующая лемма описывает некоторые свойства модифицированной статистической суммы, которые понадобятся ниже.

Лемма 3. Модифицированная статистическая сумма $\widetilde{Z}(n ; \boldsymbol{x}, \boldsymbol{y})$ обладает следующими свойствами:

а) функиия $\widetilde{Z}(n ; \boldsymbol{x}, \boldsymbol{y})$ симметрична отдельно по переменным $x_{1}, \ldots, x_{n}$ и по переменным $y_{1}, \ldots, y_{n}$;

б) функиия $\widetilde{Z}(n ; \boldsymbol{x}, \boldsymbol{y})$ является однородным полиномом по переменным $x_{i}$ и $y_{i}$ общей степени $2 n(n-1)$; для каждого фиксированного $i=1, \ldots, n$ она является полиномом по $x_{i}^{2}$ степени $n-1$ и полиномом по $y_{i}^{2}$ степени $n-1$;

в) если $y_{n}=a x_{n}$, mo

$$
\widetilde{Z}(n ; \boldsymbol{x}, \boldsymbol{y})=\sigma\left(a^{2}\right) \prod_{i=1}^{n-1}\left[\left(a y_{i}^{2}-\bar{a} x_{n}^{2}\right)\left(a y_{n}^{2}-\bar{a} x_{i}^{2}\right)\right] \widetilde{Z}\left(n-1 ; \boldsymbol{x} \backslash x_{n}, \boldsymbol{y} \backslash y_{n}\right),
$$

где $\boldsymbol{x} \backslash x_{n}=\left(x_{1}, \ldots, x_{n-1}\right) u \boldsymbol{y} \backslash y_{n}=\left(y_{1}, \ldots, y_{n-1}\right)$.

ДокАЗАТЕЛЬСтво. Утверждение "а" данной леммы следует из симметрии статистической суммы $Z(n ; \boldsymbol{x}, \boldsymbol{y})$. Утверждение "б” фактически очевидно. Рекуррентное соотношение (2) следует из соответствующего рекуррентного соотношения для статистической суммы $Z(n ; \boldsymbol{x}, \boldsymbol{y})$, которое гласит, что если $y_{n}=a x_{n}$, то

$$
Z(n ; \boldsymbol{x}, \boldsymbol{y})=\sigma\left(a^{2}\right) \prod_{i=1}^{n-1}\left[\sigma\left(a \bar{x}_{n} y_{i}\right) \sigma\left(a \bar{x}_{i} y_{n}\right)\right] Z\left(n-1 ; \boldsymbol{x} \backslash x_{n}, \boldsymbol{y} \backslash y_{n}\right) .
$$

Доказательство упомянутых свойств статистической суммы $Z(n ; \boldsymbol{x}, \boldsymbol{y})$ может быть найдено, например, в работе [3]. 
При вычислении модифицированной статистической суммы $\widetilde{Z}(n ; \boldsymbol{x}, \boldsymbol{y})$ удобно умножить вес вершины, соответствующей нулевому матричному элементу матрицы чередующихся знаков и находящейся на пересечении горизонтальной линии со спектральным параметром $x_{i}$ и вертикальной линии со спектральным параметром $y_{j}$, на $x_{i} y_{j}$. При этом множитель $x_{i} y_{j}$ извлекается из произведения, входящего как множитель в определение модифицированной статистической суммы. Следовательно, мы полагаем, что нулевой матричный элемент, соответствующий вершине третьего или четвертого типов, дает вклад $a x_{i}^{2}-\bar{a} y_{j}^{2}$ в соответствующий член модифицированной статистической суммы и что нулевой матричный элемент, соответствующий вершине пятого или шестого типов, дает вклад $a y_{j}^{2}-\bar{a} x_{i}^{2}$.

Выделяя в $\widetilde{Z}(n ; \boldsymbol{x}, \boldsymbol{y})$ член максимальной степени по переменной $x_{i}$, мы пишем

$$
\widetilde{Z}(n ; \boldsymbol{x}, \boldsymbol{y})=\left[\prod_{i=1}^{n} x_{i}^{2(n-1)}\right] C(n)+\cdots .
$$

Лемма 4. Коэфбициент $C(n)$ задается формулой

$$
C(n)=\prod_{i=1}^{n} \sigma\left(a^{2 i}\right)
$$

ДоКАЗАТЕЛЬСтво. Для того чтобы найти член максимальной степени по переменной $x_{i}$, надо брать, как легко убедиться, только такие состояния квадратного льда, которые имеют ровно один единичный матричный элемент в каждом столбце соответствующей матрицы чередующихся знаков. Лишь в этом случае все параметры $y_{i}$ из множителя, входящего в определение модифицированной статистической суммы, могут быть поглощены весами вершин. Матрицы чередующихся знаков, которые имеют только один единичный матричный элемент в каждом столбце, являются матрицами перестановок. Далее, из леммы 2 следует, что вклад в член максимальной степени по переменной $x_{i}$ от состояния, соответствующего перестановке $s$, равен

$$
\left[\prod_{i=1}^{n} x_{i}^{2(n-1)}\right] \sigma^{n}\left(a^{2}\right) a^{2 \operatorname{inv}(s)}(-\bar{a})^{n(n-1)-2 \operatorname{inv}(s)}=\left[\prod_{i=1}^{n} x_{i}^{2(n-1)}\right] \sigma^{n}\left(a^{2}\right) a^{-n(n-1)} a^{4 \operatorname{inv}(s)} .
$$

Отсюда получаем

$$
C(n)=\sigma^{n}\left(a^{2}\right) a^{-n(n-1)} \sum_{s \in S_{n}} a^{4 \operatorname{inv}(s)} .
$$

Принимая во внимание равенство (1), после некоторых элементарных преобразований приходим к соотношению (4).

Пойдем дальше и рассмотрим члены в $\widetilde{Z}(n ; \boldsymbol{x}, \boldsymbol{y})$, которые дают максимальную полную степень по всем переменным $x_{i}$ за исключением переменной $x_{n}$ и не содержат всех переменных $y_{i}$ за исключением переменной $y_{n}$. Выделяя эти члены, мы пишем

$$
\widetilde{Z}(n ; \boldsymbol{x}, \boldsymbol{y})=\left[\prod_{i=1}^{n-1} x_{i}^{2(n-1)}\right] S\left(n ; x_{n}, y_{n}\right)+\cdots
$$


Лемма 5. Полином $S\left(n ; x_{n}, y_{n}\right)$ имеет вид

$$
S\left(n ; x_{n}, y_{n}\right)=\left[\prod_{i=1}^{n-1} \sigma\left(a^{2 i}\right)\right]\left(\sigma\left(a^{2 n}\right) x_{n}^{2(n-1)}-\sigma\left(a^{2(n-1)}\right) x_{n}^{2(n-2)} y_{n}^{2}\right)
$$

ДокАзАтельство. Так как мы ищем члены, которые не содержат всех переменных $y_{i}$ за исключением переменной $y_{n}$, то мы можем рассматривать только те состояния, которые имеют лишь один единичный матричный элемент в каждом из первых $n-1$ столбцов. По определению матрица чередующихся знаков имеет лишь одну единицу и в последнем столбце тоже. Первые $n-1$ строк такой матрицы дают требуемую степень по переменным $x_{i}$. Вес вершины в последней строке равен либо $a y_{j}^{2}-\bar{a} x_{n}^{2}$, либо $a x_{n}^{2}-\bar{a} y_{j}^{2}$. В этой строке есть лишь один единичный матричный элемент. Если он находится в последнем столбце, то мы имеем степень $2(n-1)$ по переменной $x_{n}$ и нулевую степень по переменной $y_{n}$. Если же единичный матричный элемент находится не в последнем столбце, то мы имеем вклад, пропорциональный либо $x_{n}^{2(n-1)}$, либо $x_{n}^{2(n-2)} y_{n}^{2}$ в зависимости от того, какое слагаемое мы возьмем из веса последней вершины в строке (этот вес равен $a x_{n}^{2}-\bar{a} y_{n}^{2}$ ). Таким образом, мы приходим к выводу, что полином $S\left(n ; x_{n}, y_{n}\right)$ имеет вид

$$
S\left(n ; x_{n}, y_{n}\right)=C(n) x_{n}^{2(n-1)}+D(n) x_{n}^{2(n-2)} y_{n}^{2} .
$$

Из рекуррентного соотношения (2) получаем

$$
C(n)+a^{2} D(n)=\sigma\left(a^{2}\right) a^{-2(n-1)} C(n-1) .
$$

Принимая во внимание равенство (4), приходим к соотношению

$$
D(n)=-\left[\prod_{i=1}^{n-1} \sigma\left(a^{2 i}\right)\right] \sigma\left(a^{2(n-1)}\right)
$$

которое обеспечивает справедливость равенства (5).

\section{3. МОДЕЛЬ КВАДРАТНОГО ЛЬДА, СВЯЗАННАЯ С МАТРИЦАМИ ЧЕРЕДУЮЩИХСЯ ЗНАКОВ, СИММЕТРИЧНЫМИ ОТНОСИТЕЛЬНО ПОВОРОТА НА $180^{\circ}$}

3.1. Матрицы чередующихся знаков, симметричные относительно поворота на $180^{\circ}$. Будем говорить, что матрица чередующихся знаков $A$ является симметричной относительно поворота на $180^{\circ}$, если

$$
(A)_{n+1-i, n+1-j}=(A)_{i, j} .
$$

Матрица перестановки $\Sigma$, ассоциированная с перестановкой $s \in S_{n}$, симметрична относительно поворота на $180^{\circ}$ тогда и только тогда, когда

$$
s(n+1-i)=n+1-s(i), \quad i=1, \ldots, n .
$$


Обозначим множество всех перестановок, ассоциированных с $(n \times n)$-матрицами перестановок, симметричными относительно поворота на $180^{\circ}$, через $S_{n}^{\mathrm{HT}}$ и введем производящую функцию

$$
\Phi_{\mathrm{HT}}(n ; z)=\sum_{s \in S_{n}^{\mathrm{HT}}} z^{\mathrm{inv}(s)}=\sum_{k=0}^{n(n-1) / 2} I_{\mathrm{HT}}(n ; k) z^{k},
$$

где $I_{\mathrm{HT}}(n ; k)$ - число элементов $S_{n}^{\mathrm{HT}}$ с $k$ инверсиями.

Лемма 6. Справедливы равенства

$$
\begin{array}{r}
\Phi_{\mathrm{HT}}(2 m+1 ; z)=\left[\prod_{i=1}^{m}\left(1+z^{2 i+1}\right)\right] \Phi\left(m ; z^{2}\right), \\
\Phi_{\mathrm{HT}}(2 m ; z)=\left[\prod_{i=1}^{m}\left(1+z^{2 i-1}\right)\right] \Phi\left(m ; z^{2}\right) .
\end{array}
$$

Доказательство. Равенство (6) выполняется для $m=0$. Предположим, что оно выполняется для некоторого $m=k \geqslant 0$. Пусть $s$ - произвольный элемент $S_{2 k+1}^{\mathrm{HT}}$. Отождествим его с перестановкой $s^{\prime}$ алфавита $\{2,3, \ldots, 2 k+2\}$, определяемой равенством

$$
s^{\prime}(i)=s(i-1)+1,
$$

и представим $s$ как слово $s^{\prime}(2) s^{\prime}(3) \ldots s^{\prime}(2 k+2)$. Если мы вставим буквы 1 и $2 k+3$ в это слово симметричным образом, то мы получим слово, представляющее элемент из $S_{2 k+3}^{\mathrm{HT}}$. Ясно, что таким способом можно получить все элементы из $S_{2 k+3}^{\mathrm{HT}}$, причем каждый элемент получается только одним способом. Если мы вставим букву 1 перед словом, а букву $2 k+3$ - после него, то получим элемент $S_{2 k+3}^{\mathrm{HT}}$, имеющий то же самое число инверсий, что и исходный элемент $S_{2 k+1}^{\mathrm{HT}}$. Это дает вклад, равный $\Phi_{\mathrm{HT}}(2 k+1 ; z)$, в производящую функцию $\Phi_{\mathrm{HT}}(2 k+3 ; z)$. Если мы вставим букву 1 во вторую позицию и букву $2 k+3$ - в предпоследнюю, то получим элемент $S_{2 k+3}^{\mathrm{HT}}$, имеющий число инверсий на два больше, чем исходный элемент $S_{2 k+1}^{\mathrm{HT}}$. Это дает вклад, равный $\Phi_{\mathrm{HT}}(2 k+1 ; z) z^{2}$. Продолжая эту процедуру, мы исчерпаем все элементы из $S_{2 k+3}^{\mathrm{HT}}$. Заметим, что число инверсий увеличивается на три, когда мы проходим через середину слова. Таким образом, мы имеем

$$
\begin{aligned}
\Phi_{\mathrm{HT}}(2 k+3 ; z) & =\Phi_{\mathrm{HT}}(2 k+1 ; z)\left(1+z^{2}+\cdots+z^{2 k}+z^{2 k+3}+\cdots+z^{4 k+3}\right)= \\
& =\Phi_{\mathrm{HT}}(2 k+1 ; z)\left(1+z^{2 k+3}\right)\left(1+z^{2}+\cdots+z^{2 k}\right) .
\end{aligned}
$$

Из этого равенства следует, что соотношение (6) справедливо для $m=k+1$. Следовательно, оно справедливо и для всех $m \geqslant 0$. Равенство (7) может быть доказано аналогично.

3.2. Модель квадратного льда для матриц четного порядка. Метод построения моделей квадратного льда, соответствующих матрицам чередующихся знаков, обладающих некоторой симметрией, был предложен Купербергом [3]. В этом 
методе рассматривается такое подмножество вершин состояния, соответствующего всей матрице чередующихся знаков, которое однозначно ее определяет, и затем подходящим образом задаются спектральные параметры. Куперберг рассмотрел модель квадратного льда, соответствующую матрицам чередующихся знаков, обладающих симметрией относительно поворота на $180^{\circ}$ и имеющих четный порядок. Структуру состояния и спецификацию спектральных параметров модели можно понять из примера, приведенного на рис. $9^{2)}$.

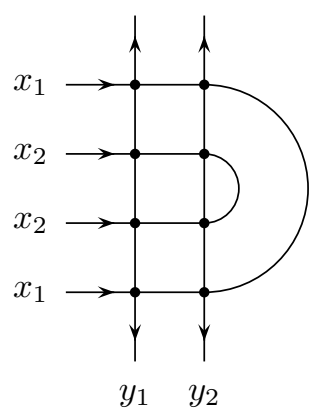

Рис. 9. Квадратный лед с границей четного размера, симметричной относительно поворота на $180^{\circ}$.

Имеется следующий очевидный аналог леммы 2.

ЛЕмма 7. Для состояния квадратного льда с гранищей четного размера, симметричной относителъно поворота на $180^{\circ}$, которое соответствует матрице перестановки, ассоциированной с перестановкой $s \in S_{2 m}^{\mathrm{HT}}$, общее число вериин третъего типа и четвертого типа равно $\operatorname{inv}(s)$, а общее число вериин пятого типа и шестого типа равно $m(2 m-1)-\operatorname{inv}(s)$.

Будем обозначать статистическую сумму квадратного льда с границей четного размера, симметричной относительно поворота на $180^{\circ}$, через $Z_{\mathrm{HT}}(2 m ; \boldsymbol{x}, \boldsymbol{y})^{3)}$. Эта функция является полиномом Лорана по переменным $x_{i}$ и $y_{i}$. Введем модифицированную статистическую сумму

$$
\widetilde{Z}_{\mathrm{HT}}(2 m ; \boldsymbol{x}, \boldsymbol{y})=\left[\prod_{i=1}^{m} x_{i}^{2 m-1} y_{i}^{2 m-1}\right] Z_{\mathrm{HT}}(2 m ; \boldsymbol{x}, \boldsymbol{y}) .
$$

Лемма 8. Модифицированная статистическая сумма $\widetilde{Z}_{\mathrm{HT}}(2 m ; \boldsymbol{x}, \boldsymbol{y})$ обладает следуюшими свойствами:

а) функиия $\widetilde{Z}_{\mathrm{HT}}(2 m ; \boldsymbol{x}, \boldsymbol{y})$ симметрична в отдельности по переменным $x_{1}, \ldots$ $\ldots, x_{m}$ и по переменным $y_{1}, \ldots, y_{m}$;

${ }^{2)}$ В действительности Куперберг рассмотрел два варианта модели, различающихся способом задания спектральных параметров. Для наших целей достаточно рассмотреть лишь один вариант.

3) Куперберг в работе [3] рассматривал матрицы чередующихся знаков, обладающие симметрией относительно поворота на $180^{\circ}$ и имеющие только четный порядок. Он обозначал статистическую сумму $Z_{\mathrm{HT}}(2 m ; \boldsymbol{x}, \boldsymbol{y})$ как $Z_{\mathrm{HT}}(m ; \boldsymbol{x}, \boldsymbol{y})$. 
б) функиия $\widetilde{Z}_{\mathrm{HT}}(2 m ; \boldsymbol{x}, \boldsymbol{y})$ является однородным полиномом по переменным $x_{i}$ и $y_{i}$ общей степени $2 m(2 m-1)$; для каждого биксированного $i=1, \ldots, m$ она является полиномом по $x_{i}^{2}$ степени $2 m-1$ и полиномом по $y_{i}^{2}$ степени $2 m-1$;

в) если $y_{m}=a x_{m}$, mo

$$
\begin{aligned}
\widetilde{Z}_{\mathrm{HT}}(2 m ; \boldsymbol{x}, \boldsymbol{y})= & \sigma^{2}\left(a^{2}\right) x_{m} y_{m} \times \\
& \times \prod_{i=1}^{m-1}\left[\left(a y_{i}^{2}-\bar{a} x_{m}^{2}\right)^{2}\left(a y_{m}^{2}-\bar{a} x_{i}^{2}\right)^{2}\right] \widetilde{Z}_{\mathrm{HT}}\left(2 m-2 ; \boldsymbol{x} \backslash x_{m}, \boldsymbol{y} \backslash y_{m}\right) .
\end{aligned}
$$

ДокАзАТЕЛЬСтво. Указанные свойства модифицированной статистической суммы $\widetilde{Z}_{\mathrm{HT}}(2 m ; \boldsymbol{x}, \boldsymbol{y})$ следуют из соответствующих свойств статистической суммы $Z_{\mathrm{HT}}(2 m ; \boldsymbol{x}, \boldsymbol{y})[3]$.

Выделяя из $\widetilde{Z}_{\mathrm{HT}}(2 m ; \boldsymbol{x}, \boldsymbol{y})$ член максимальной степени по переменным $x_{i}$, мы пишем

$$
\widetilde{Z}_{\mathrm{HT}}(2 m ; \boldsymbol{x}, \boldsymbol{y})=\left[\prod_{i=1}^{m} x_{i}^{2(2 m-1)}\right] C_{\mathrm{HT}}(2 m)+\cdots .
$$

Лемма 9. Коэфбициент $C_{\mathrm{HT}}(2 m)$ задается формулой

$$
C_{\mathrm{HT}}(2 m)=\prod_{i=1}^{2 m} \sigma\left(a^{i}\right)
$$

ДоКАЗАТЕЛЬСтво. Понятно, что лишь состояния, соответствующие матрицам перестановок, дают вклад в член максимальной степени по переменным $x_{i}$. Используя ту же аргументацию, что и при доказательстве леммы 4 , и принимая во внимание лемму 7 , мы видим, что вклад в состояние, соответствующее перестановке $s$, равен

$$
\begin{gathered}
{\left[\prod_{i=1}^{m} x_{i}^{2(2 m-1)}\right] \sigma^{m}\left(a^{2}\right) a^{\operatorname{inv}(s)}(-\bar{a})^{m(2 m-1)-\operatorname{inv}(s)}=} \\
=\left[\prod_{i=1}^{m} x_{i}^{2(2 m-1)}\right] \sigma^{m}\left(a^{2}\right)(-a)^{-m(2 m-1)}\left(-a^{2}\right)^{\operatorname{inv}(s)} .
\end{gathered}
$$

Таким образом,

$$
C_{\mathrm{HT}}(2 m)=(-1)^{m} \sigma^{m}\left(a^{2}\right) a^{-m(2 m-1)} \sum_{s \in S_{2 m}^{\mathrm{HT}}}\left(-a^{2}\right)^{\operatorname{inv}(s)} .
$$

Используя соотношение (7), приходим к равенству (8).

Выделяя члены $\widetilde{Z}_{\mathrm{HT}}(2 m ; \boldsymbol{x}, \boldsymbol{y})$, имеющие максимальную общую степень по всем переменным $x_{i}$, кроме переменной $x_{m}$, и не содержащие никаких переменных $y_{i}$, кроме $y_{m}$, мы пишем

$$
\widetilde{Z}_{\mathrm{HT}}(2 m ; \boldsymbol{x}, \boldsymbol{y})=\left[\prod_{i=1}^{m-1} x_{i}^{2(2 m-1)}\right] S_{\mathrm{HT}}\left(2 m ; x_{m}, y_{m}\right)+\cdots .
$$


Чтобы найти полином $S_{\mathrm{HT}}\left(2 m ; x_{m}, y_{m}\right)$, используем тот факт, что, как доказано Купербергом [3], статистическая сумма $Z_{\mathrm{HT}}(2 m ; \boldsymbol{x}, \boldsymbol{y})$ является произведением двух полиномов Лорана,

$$
Z_{\mathrm{HT}}(2 m ; \boldsymbol{x}, \boldsymbol{y})=Z(m ; \boldsymbol{x}, \boldsymbol{y}) Z_{\mathrm{HT}}^{(2)}(2 m ; \boldsymbol{x}, \boldsymbol{y}) .
$$

Следовательно, можно написать

$$
\widetilde{Z}_{\mathrm{HT}}(2 m ; \boldsymbol{x}, \boldsymbol{y})=\widetilde{Z}(m ; \boldsymbol{x}, \boldsymbol{y}) \widetilde{Z}_{\mathrm{HT}}^{(2)}(2 m ; \boldsymbol{x}, \boldsymbol{y}) .
$$

ЛЕмма 10. Функция $\widetilde{Z}_{\mathrm{HT}}^{(2)}(2 m ; \boldsymbol{x}, \boldsymbol{y})$ является однородным полиномом по переменным $x_{i}$ и $y_{i}$ степени $2 m^{2}$. Для каждого фиксированного $i=1, \ldots, m$ она является полиномом по $x_{i}^{2}$ степени $m$ и полиномом по $y_{i}^{2}$ степени $m$. Если $y_{m}=a x_{m}$, mo

$$
\begin{aligned}
\widetilde{Z}_{\mathrm{HT}}^{(2)}(2 m ; \boldsymbol{x}, \boldsymbol{y})=\sigma\left(a^{2}\right) x_{m} y_{m} \times & \\
& \times \prod_{i=1}^{m-1}\left[\left(a y_{i}^{2}-\bar{a} x_{m}^{2}\right)\left(a y_{m}^{2}-\bar{a} x_{i}^{2}\right)\right] \widetilde{Z}_{\mathrm{HT}}^{(2)}\left(2(m-1) ; \boldsymbol{x} \backslash x_{m}, \boldsymbol{y} \backslash y_{m}\right) .
\end{aligned}
$$

ДокАзАТЕЛЬСтво. Лемму можно доказать, используя соотношение (9) и леммы 8 и 3.

Лемма 11. Полином $S_{\mathrm{HT}}\left(2 m ; x_{m}, y_{m}\right)$ имеет вид

$$
S_{\mathrm{HT}}\left(2 m ; x_{m}, y_{m}\right)=S\left(m ; x_{m}, y_{m}\right) S_{\mathrm{HT}}^{(2)}\left(2 m, x_{m}, y_{m}\right),
$$

где $S\left(m ; x_{m}, y_{m}\right)$ задается соотношением (5),

$$
S_{\mathrm{HT}}^{(2)}\left(2 m ; x_{m}, y_{m}\right)=\left[\prod_{i=1}^{m-1} \sigma\left(a^{2 i-1}\right)\right]\left(\sigma\left(a^{2 m-1}\right) x_{m}^{2 m}+\sigma\left(a^{2 m-3}\right) x_{m}^{2(m-1)} y_{m}^{2}\right) .
$$

ДокАЗАТЕЛЬСтво. Рассмотрим состояние квадратного льда с границей, симметричной относительно поворота на $180^{\circ}$, дающее нетривиальный вклад в полином $S_{\mathrm{HT}}\left(2 m ; x_{m}, y_{m}\right)$. Очевидно, что для такого состояния каждая линия со спектральным параметром $y_{j}, j=1, \ldots, m-1$, может иметь лишь одну вершину первого или второго типов. То же самое справедливо и для каждой линии со спектральным параметром $x_{i}, i=1, \ldots, m-1$. Для линии со спектральным параметром $x_{m}$ мы имеем две возможности. Либо она имеет лишь одну вершину первого или второго типов, либо она имеет две вершины первого типа и одну - второго. В последнем случае линия со спектральным параметром $y_{m}$ также имеет две вершины первого типа и одну - второго. Анализируя все эти возможности, можно заключить, что полином $S_{\mathrm{HT}}\left(2 m ; x_{m}, y_{m}\right)$ имеет вид

$$
S_{\mathrm{HT}}\left(2 m ; x_{m}, y_{m}\right)=C_{\mathrm{HT}}(2 m) x_{m}^{2(2 m-1)}+D_{\mathrm{HT}}(2 m) x_{m}^{2(2 m-2)} y_{m}^{2}+E_{\mathrm{HT}}(2 m) x_{m}^{2(2 m-3)} y_{m}^{4} \text {. }
$$

Из равенства (9) следует, что полином $S_{\mathrm{HT}}\left(2 m ; x_{m}, y_{m}\right)$ может быть представлен в виде (11) при некотором полиноме $S_{\mathrm{HT}}^{(2)}\left(2 m ; x_{m}, y_{m}\right)$. Следовательно, имея в виду (5), мы можем заключить, что

$$
S_{\mathrm{HT}}^{(2)}\left(2 m ; x_{m}, y_{m}\right)=C_{\mathrm{HT}}^{(2)}(2 m) x_{m}^{2 m}+D_{\mathrm{HT}}^{(2)}(2 m) x_{m}^{2(m-1)} y_{m}^{2} .
$$

2 Теоретическая и математическая физика, т. 148, № 3, 2006 г. 
Используя очевидное равенство

$$
C_{\mathrm{HT}}(2 m)=C(m) C_{\mathrm{HT}}^{(2)}(2 m),
$$

получаем соотношение

$$
C_{\mathrm{HT}}^{(2)}(2 m)=\prod_{i=1}^{m} \sigma\left(a^{2 i-1}\right) .
$$

Рекуррентное соотношение (10) дает

$$
C_{\mathrm{HT}}^{(2)}(2 m)+a^{2} D_{\mathrm{HT}}^{(2)}(2 m)=\sigma\left(a^{2}\right) a^{-2 m+3} C_{\mathrm{HT}}^{(2)}(2(m-1)) .
$$

Принимая во внимание равенство (8), получаем соотношение

$$
D_{\mathrm{HT}}^{(2)}(2 m)=-\left[\prod_{i=1}^{m-1} \sigma\left(a^{2 i-1}\right)\right] \sigma\left(a^{2 m-3}\right),
$$

которое имеет следствием равенство (12).

3.3. Модель квадратного льда для матриц нечетного порядка. Перейдем теперь к матрицам чередующихся знаков нечетного порядка, симметричным относительно поворота на $180^{\circ}$. Полезно иметь в виду, что центральный элемент такой матрицы равен 1 или -1. Структуру состояния и спецификацию спектральных параметров можно понять из примера, приведенного на рис. 10. Крест обозначает смену спектрального параметра, ассоциированого с линией. При этом направление стрелок должно сохраняться. Следующая лемма очевидна.

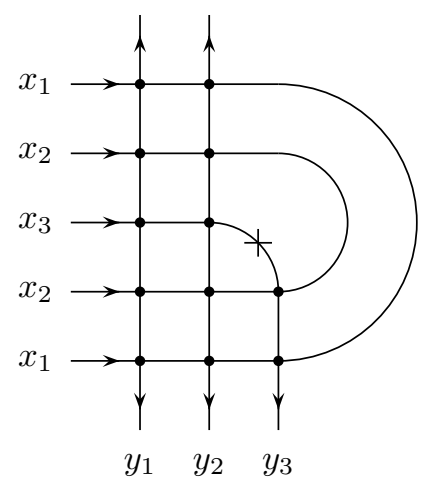

Рис. 10. Квадратный лед с границей нечетного размера, симметричной относительно поворота на $180^{\circ}$.

ЛЕмма 12. Для состояния квадратного льда с границей нечетного размера, симметричной относительно поворота на $180^{\circ}$, которое соответствует матрице перестановки, ассочиированной с перестановкой $s \in S_{2 m+1}^{\mathrm{HT}}$, полное число вериин третьего типа и четвертого типа равно $\operatorname{inv}(s)$, а полное число вершин пятого mипа и шестого типа равно $m(2 m+1)-\operatorname{inv}(s)$. 
Статистическая сумма $Z_{\mathrm{HT}}(2 m+1 ; \boldsymbol{x}, \boldsymbol{y})$ является полиномом Лорана по переменным $x_{i}$ и $y_{i}$. Удобно ввести модифицированную статистическую сумму

$$
\widetilde{Z}_{\mathrm{HT}}(2 m+1 ; \boldsymbol{x}, \boldsymbol{y})=\left[\prod_{i=1}^{m} x_{i}^{2 m} y_{i}^{2 m}\right] x_{m+1}^{m} y_{m+1}^{m} Z_{\mathrm{HT}}(2 m+1 ; \boldsymbol{x}, \boldsymbol{y}),
$$

которая является полиномом по спектральным параметрам.

ЛЕмма 13. Статистическая сумма $Z_{\mathrm{HT}}(2 m+1 ; \boldsymbol{x}, \boldsymbol{y})$ является симметричной бункиией отдельно по переменным $x_{1}, \ldots, x_{m}$ и по переменным $y_{1}, \ldots, y_{m}$. Если $y_{1}=a x_{1}, m o$

$$
\begin{aligned}
Z_{\mathrm{HT}}(2 m+1 ; \boldsymbol{x}, \boldsymbol{y})= & \sigma^{2}\left(a^{2}\right) \sigma\left(a \bar{x}_{1} y_{m+1}\right) \sigma\left(a \bar{x}_{m+1} y_{1}\right) \times \\
& \times \prod_{i=2}^{m}\left[\sigma^{2}\left(a \bar{x}_{1} y_{i}\right) \sigma^{2}\left(a \bar{x}_{i} y_{1}\right)\right] Z_{\mathrm{HT}}\left(2 m-1 ; \boldsymbol{x} \backslash x_{1}, \boldsymbol{y} \backslash y_{1}\right) .
\end{aligned}
$$

ДоКАЗАТЕЛЬСтво. Для того чтобы доказать симметрию статистической суммы $Z_{\mathrm{HT}}(2 m+1 ; \boldsymbol{x}, \boldsymbol{y})$ по переменным $x_{1}$ и $x_{2}$, умножим ее на $\sigma(a z)$. Полученному выражению может быть сопоставлен граф в левой части равенства, приведенного на рис. 11. Пусть $z=a \bar{x}_{1} x_{2}$, тогда, используя уравнение Янга-Бакстера (см. рис. 4),
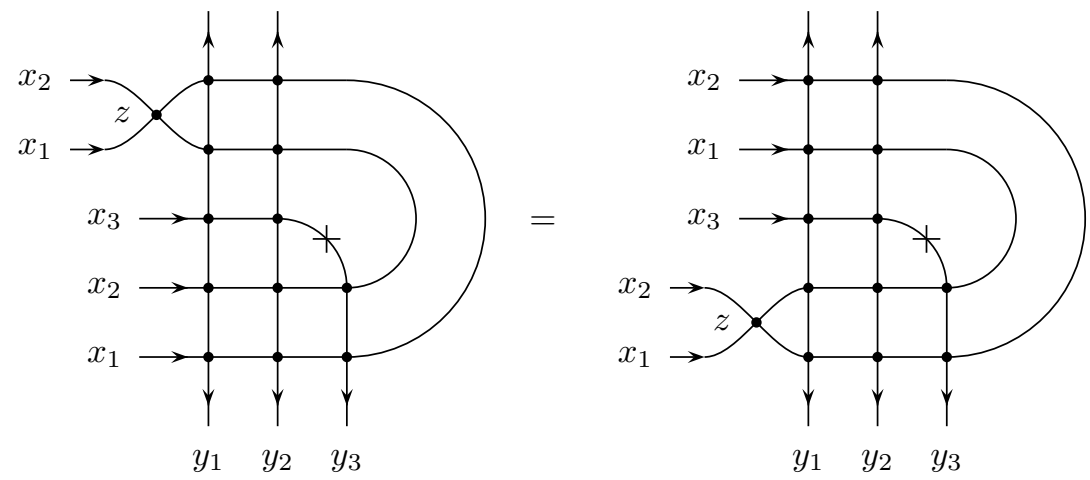

Рис. 11. Доказательство симметричности $Z_{\mathrm{HT}}(3 ; \boldsymbol{x}, \boldsymbol{y})$ по $x_{1}$ и $x_{2}$.

можно переместить пересечение в позицию, показанную на графе в правой части равенства. Это доказывает симметричность по $x_{1}$ и $x_{2}$. Другие переменные исследуются таким же способом.

Чтобы доказать рекуррентное соотношение (14), замечаем, что если $y_{1}=a x_{1}$, то лишь состояния с вершиной первого типа в верхнем левом углу дают не равный нулю вклад в статистическую сумму. При этом четырехвалентные вершины на границе графа фиксируются, см. рис. 12. Они порождают все множители, кроме последнего в правой части (14), а оставшиеся вершины порождают последний множитель.

Лемма 14. Модифицированная статистическая сумма $\widetilde{Z}_{\mathrm{HT}}(2 m+1 ; \boldsymbol{x}, \boldsymbol{y})$ обладает следующими свойствами: 


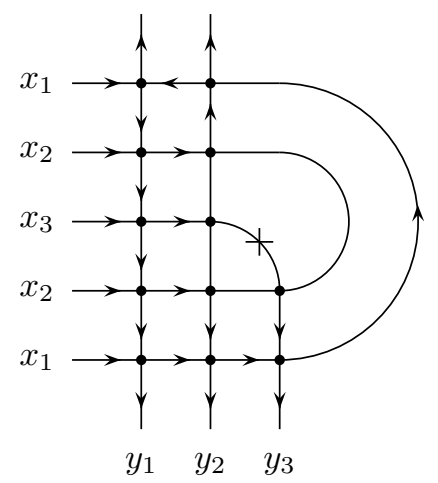

Рис. 12. Доказательство рекуррентного соотношения (14).

а) функиия $\widetilde{Z}_{\mathrm{HT}}(2 m+1 ; \boldsymbol{x}, \boldsymbol{y})$ симметрична отдельно по переменным $x_{1}, \ldots, x_{m}$ и по переменным $y_{1}, \ldots, y_{m}$;

б) функиия $\widetilde{Z}_{\mathrm{HT}}(2 m+1 ; \boldsymbol{x}, \boldsymbol{y})$ является однородным полиномом по переменным $x_{i}$ и $y_{i}$ степени $2 m(2 m+1)$; для каждого фиксированного $i=1, \ldots, m$ она является полиномом по $x_{i}^{2}$ степени $2 m$ и полиномом по $y_{i}^{2}$ степени $2 m$; она также является полиномом степени $2 m$ по $x_{m+1}$ и полиномом степени $2 m$ по $y_{m+1}$;

в) если $y_{m}=a x_{m}$, mo

$$
\begin{aligned}
\widetilde{Z}_{\mathrm{HT}}(2 m+ & 1 ; \boldsymbol{x}, \boldsymbol{y})=\sigma^{2}\left(a^{2}\right)\left(a y_{m+1}^{2}-\bar{a} x_{m}^{2}\right)\left(a y_{m}^{2}-\bar{a} x_{m+1}^{2}\right) x_{m} y_{m} \times \\
& \times \prod_{i=1}^{m-1}\left[\left(a y_{i}^{2}-\bar{a} x_{m}^{2}\right)^{2}\left(a y_{m}^{2}-\bar{a} x_{i}^{2}\right)^{2}\right] \widetilde{Z}_{\mathrm{HT}}\left(2 m-1 ; \boldsymbol{x} \backslash x_{m}, \boldsymbol{y} \backslash y_{m}\right) .
\end{aligned}
$$

ДокАзательство. Первые два утверждения леммы очевидны. Рекуррентные соотношения (15) следуют из симметричности $Z_{\mathrm{HT}}(2 m+1 ; \boldsymbol{x}, \boldsymbol{y})$ и рекуррентных соотношений (14).

Выделяя из $\widetilde{Z}_{\mathrm{HT}}(2 m+1 ; \boldsymbol{x}, \boldsymbol{y})$ член максимальной степени по переменным $x_{i}$, мы пишем

$$
\widetilde{Z}_{\mathrm{HT}}(2 m+1 ; \boldsymbol{x}, \boldsymbol{y})=\left[\prod_{i=1}^{m} x_{i}^{4 m}\right] x_{m+1}^{2 m} C_{\mathrm{HT}}(2 m+1)+\cdots .
$$

ЛЕмма 15. Коэффиииент $C_{\mathrm{HT}}(2 m+1)$ задается формулой

$$
C_{\mathrm{HT}}(2 m+1)=\prod_{i=2}^{2 m+1} \sigma\left(a^{i}\right) .
$$

ДокАЗАтЕЛЬСтво. Лемму можно доказать тем же способом, что и лемму 9, используя лемму 12 и равенство (6).

Выделяя из $\widetilde{Z}_{\mathrm{HT}}(2 m+1 ; \boldsymbol{x}, \boldsymbol{y})$ член, имеющий максимальную степень по всем спектральным параметрам, кроме $x_{m+1}$ и $y_{m+1}$, мы пишем

$$
\widetilde{Z}_{\mathrm{HT}}(2 m+1 ; \boldsymbol{x}, \boldsymbol{y})=\left[\prod_{i=1}^{m} x_{i}^{4 m}\right] S_{\mathrm{HT}}\left(2 m+1 ; x_{m+1}, y_{m+1}\right)+\cdots
$$


Свойства модифицированных статистических сумм $\widetilde{Z}(n ; \boldsymbol{x}, \boldsymbol{y})$ и $\widetilde{Z}_{\mathrm{HT}}(2 m ; \boldsymbol{x}, \boldsymbol{y})$, описанные в леммах 3 и 8, соответственно, однозначно определяют статистические суммы посредством интерполяции Лагранжа. Это не так для модифицированной статистической суммы $\widetilde{Z}_{\text {Нт }}(2 m+1 ; \boldsymbol{x}, \boldsymbol{y})$. Однако мы можем получить недостающую информацию, найдя полином $S_{\mathrm{HT}}\left(2 m+1 ; x_{m+1}, y_{m+1}\right)$. Чтобы доказать это, нам потребуется следующая лемма.

ЛЕмма 16. Статистическая сумма $Z_{\mathrm{HT}}(2 m+1 ; \boldsymbol{x}, \boldsymbol{y})$ инвариантна относительно замен $x_{i} \rightarrow \bar{x}_{i}, y_{i} \rightarrow \bar{y}_{i}$. Для модифицированной статистической суммы имеем

$$
\widetilde{Z}_{\mathrm{HT}}(2 m+1 ; \overline{\boldsymbol{x}}, \overline{\boldsymbol{y}})=\left[\prod_{i=1}^{m} x_{i}^{-4 m} y_{i}^{-4 m}\right] x_{m+1}^{-2 m} y_{m+1}^{-2 m} \widetilde{Z}_{\mathrm{HT}}(2 m+1 ; \boldsymbol{x}, \boldsymbol{y}),
$$

где $\overline{\boldsymbol{x}}=\left(\bar{x}_{1}, \ldots, \bar{x}_{m+1}\right)$ u $\overline{\boldsymbol{y}}=\left(\bar{y}_{1}, \ldots, \bar{y}_{m+1}\right)$.

ДокАЗАтЕЛЬСтво. Рассмотрим состояние квадратного льда с границей, симметричной относительно поворота на $180^{\circ}$. Отразим соответствующий граф относительно горизонтальной линии и затем повернем половину линии со спектральным параметром $y_{m+1}$ на $180^{\circ}$. Ясно, что вес нового состояния получается из веса старого заменами $x_{i} \rightarrow \bar{x}_{i}, y_{i} \rightarrow \bar{y}_{i}$. Этот факт влечет за собой инвариантность $Z_{\mathrm{HT}}(2 m+1 ; \boldsymbol{x}, \boldsymbol{y})$ относительно этих замен. Равенство (17) следует немедленно из инвариантности $Z_{\mathrm{HT}}(2 m+1 ; \boldsymbol{x}, \boldsymbol{y})$.

ЛЕмма 17. Предположим, что две функции $\widetilde{Z}_{\mathrm{HT}}(2 m+1 ; \boldsymbol{x}, \boldsymbol{y})$ и $\widetilde{Z}_{\mathrm{HT}}^{\prime}(2 m+1 ; \boldsymbol{x}, \boldsymbol{y})$ обладают свойствами, описанными в лемме 14, и соответствующие полиномь $S_{\mathrm{HT}}\left(2 m+1 ; x_{m+1}, y_{m+1}\right)$ u $S_{\mathrm{HT}}^{\prime}\left(2 m+1 ; x_{m+1}, y_{m+1}\right)$ совпадают. Тогда $\widetilde{Z}_{\mathrm{HT}}(2 m+1 ;$ $\boldsymbol{x}, \boldsymbol{y})=\widetilde{Z}_{\mathrm{HT}}^{\prime}(2 m+1 ; \boldsymbol{x}, \boldsymbol{y})$.

ДокАЗАТЕЛЬСтво. Используя утверждения "а" и "б" леммы 14, можно видеть, что разность между $\widetilde{Z}_{\mathrm{HT}}(2 m+1 ; \boldsymbol{x}, \boldsymbol{y})$ и $\widetilde{Z}_{\mathrm{HT}}^{\prime}(2 m+1 ; \boldsymbol{x}, \boldsymbol{y})$ равна нулю при $y_{j}=a x_{i}$. Так как $\widetilde{Z}_{\mathrm{HT}}(2 m+1 ; \boldsymbol{x}, \boldsymbol{y})$ и $\widetilde{Z}_{\mathrm{HT}}^{\prime}(2 m+1 ; \boldsymbol{x}, \boldsymbol{y})$ являются полиномами по $y_{i}^{2}$, эта разность также равна нулю при $y_{j}=-a x_{i}$. Используя (17), можно получить рекуррентные соотношения (15) для случая $y_{1}=\bar{a} x_{1}$. Из этих рекуррентных соотношений (15) и утверждения "а" леммы 14 следует, что разность $\widetilde{Z}_{\mathrm{HT}}(2 m+1 ; \boldsymbol{x}, \boldsymbol{y})$ и $\widetilde{Z}_{\mathrm{HT}}^{\prime}(2 m+1 ; \boldsymbol{x}, \boldsymbol{y})$ равна нулю при $y_{j}=\bar{a} x_{i}$ и $y_{j}=-\bar{a} x_{i}$. Используя эти факты, легко получаем равенство

$$
\begin{aligned}
\widetilde{Z}_{\mathrm{HT}}(2 m+ & 1 ; \boldsymbol{x}, \boldsymbol{y})-\widetilde{Z}_{\mathrm{HT}}^{\prime}(2 m+1 ; \boldsymbol{x}, \boldsymbol{y})=\prod_{i, j=1}^{m}\left[\left(y_{i}^{2}-a^{2} x_{j}\right)\left(y_{i}^{2}-\bar{a}^{2} x_{j}\right)\right] \times \\
& \times\left(S_{\mathrm{HT}}\left(2 m+1 ; x_{m+1}, y_{m+1}\right)-S_{\mathrm{HT}}^{\prime}\left(2 m+1 ; x_{m+1}, y_{m+1}\right)\right),
\end{aligned}
$$

которое делает очевидным утверждение леммы.

3.4. Дополнительные рекуррентные соотношения. Для нахождения полинома $S_{\mathrm{HT}}\left(2 m+1 ; x_{m+1}, y_{m+1}\right)$ мы используем дополнительные рекуррентные соотношения, которым удовлетворяет статистическая сумма $Z_{\mathrm{HT}}(2 m+1 ; \boldsymbol{x}, \boldsymbol{y})$. 
ЛЕмма 18. Если $y_{m+1}=a x_{m+1}$, mo

$$
Z_{\mathrm{HT}}(2 m+1 ; \boldsymbol{x}, \boldsymbol{y})=\prod_{i=1}^{m}\left[\sigma\left(a \bar{x}_{i} y_{m+1}\right) \sigma\left(a \bar{x}_{m+1} y_{i}\right)\right] Z_{\mathrm{HT}}\left(2 m ; \boldsymbol{x} \backslash x_{m+1}, \boldsymbol{y} \backslash y_{m+1}\right) .
$$

ДокАзАтельство. Рассмотрим спектральные параметры вершин, принадлежащих центральному "треугольнику" графа, описывающего состояние квадратного льда с границей симметричной относительно поворота на 180, см. пример на рис. 13. Ясно, что если $y_{m+1}=a x_{m+1}$, то мы можем использовать уравнение Янга-Бакстера,
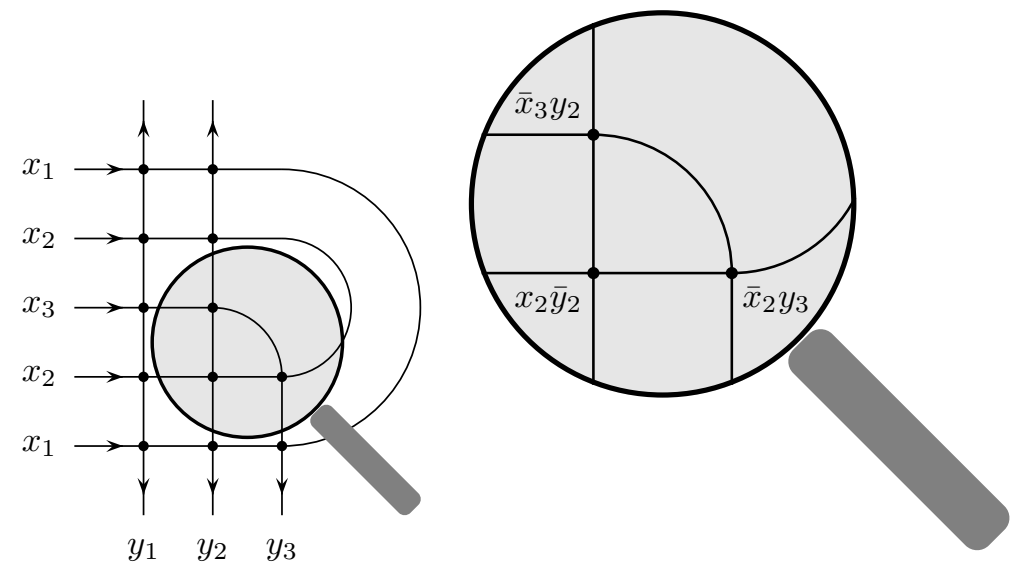

Рис. 13. Центральный “треугольник” под увеличительным стеклом.

приведенное на рис. 4 , и передвинуть линию со спектральными параметрами $x_{m+1}$ и $y_{m+1}$ к границе. Пример такого процесса приведен на рис. 14 . Отметим, что в середине процесса веса перемещаемых вершин определяются не по стандартным правилам. В действительности параметры $x_{m+1}$ и $y_{m+1}$ меняются ролями. В конечном состоянии мы можем использовать стандартные правила, если поменяем местами спектральные параметры $x_{m+1}$ и $y_{m+1}$, как это сделано на рис. 14 . Последний граф на рис. 14 доказывает утверждение леммы.

Следующая лемма является прямым следствием предыдущей.

ЛЕмма 19. Если $y_{m+1}=a x_{m+1}$, mo

$$
\widetilde{Z}_{\mathrm{HT}}(2 m+1 ; \boldsymbol{x}, \boldsymbol{y})=\prod_{i=1}^{m}\left[\left(a y_{m+1}^{2}-\bar{a} x_{i}^{2}\right)\left(a y_{i}^{2}-\bar{a} x_{m+1}^{2}\right)\right] \widetilde{Z}_{\mathrm{HT}}\left(2 m ; \boldsymbol{x} \backslash x_{m+1}, \boldsymbol{y} \backslash y_{m+1}\right) \text {. }
$$

Теперь мы можем найти полином $S_{\mathrm{HT}}\left(2 m+1 ; x_{m+1}, y_{m+1}\right)$.

ЛЕмма 20. Полином $S_{\mathrm{HT}}\left(2 m+1 ; x_{m+1}, y_{m+1}\right)$ имеет вид

$$
S_{\mathrm{HT}}\left(2 m+1 ; x_{m+1}, y_{m+1}\right)=\left[\prod_{i=2}^{2 m} \sigma\left(a^{i}\right)\right]\left(\sigma\left(a^{2 m+1}\right) x_{m+1}^{2 m}-\sigma\left(a^{2 m}\right) x_{m+1}^{2 m-1} y_{m+1}\right) .
$$



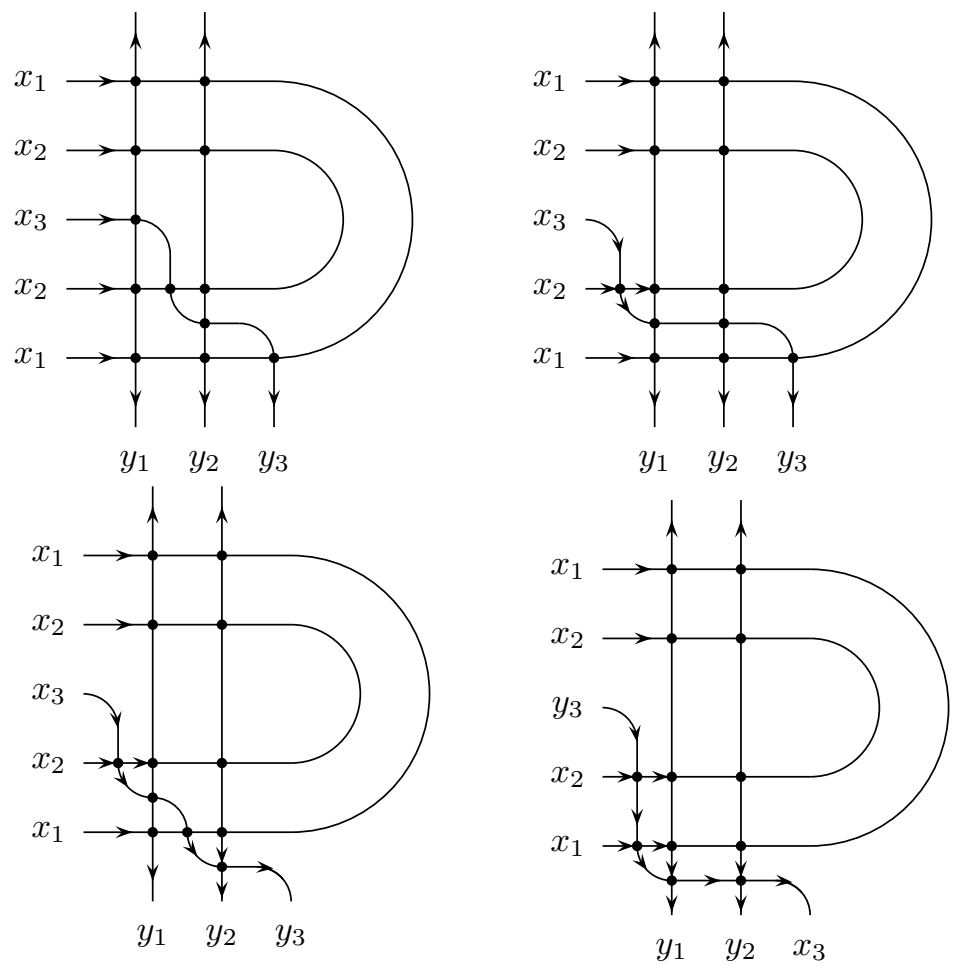

Рис. 14. Доказательство рекуррентных соотношений (18).

ДокАзАтельство. Рассмотрим какое-нибудь состояние квадратного льда с границей, симметричной относительно поворота на $180^{\circ}$, дающее нетривиальный вклад в полином $S_{\mathrm{HT}}\left(2 m+1 ; x_{m}, y_{m}\right)$. Очевидно, что для такого состояния каждая линия со спектральным параметром $y_{j}, j=1, \ldots, m$, может иметь лишь одну вершину первого или второго типов. То же самое верно для каждой линии со спектральным параметром $x_{i}, i=1, \ldots, m$. Для каждой линии со спектральными параметрами $x_{m+1}$ и $y_{m+1}$ мы имеем две возможности. Она или не имеет вершин первого и второго типов, или имеет две вершины первого типа. В первом случае имеется одна скрытая вершина первого типа в точке поворота, а во втором случае там же имеется скрытая вершина второго типа. Можно убедится в том, что полином $S_{\mathrm{HT}}\left(2 m+1 ; x_{m+1}, y_{m+1}\right)$ имеет вид

$$
S_{\mathrm{HT}}\left(2 m+1 ; x_{m+1}, y_{m+1}\right)=C_{\mathrm{HT}}(2 m+1) x_{m+1}^{2 m}+D_{\mathrm{HT}}(2 m+1) x_{m+1}^{2 m-1} y_{m+1} .
$$

Из рекуррентных соотношений (19) следует, что

$$
C_{\mathrm{HT}}(2 m+1)+a D_{\mathrm{HT}}(2 m+1)=a^{-2 m} C_{\mathrm{HT}}(2 m) .
$$

Используя (16), получаем равенство

$$
D_{\mathrm{HT}}(2 m+1)=-\left[\prod_{i=2}^{2 m} \sigma\left(a^{i}\right)\right] \sigma\left(a^{2 m}\right),
$$


которое немедленно приводит к (19).

\section{5. Главная теорема.}

Теорема 1. Статистическая сумма для модели квадратного льда с границей, симметричной относителъно поворота на $180^{\circ}$, может быть представлена в виде

$$
\begin{aligned}
Z_{\mathrm{HT}}(2 m+1 ; \boldsymbol{x}, \boldsymbol{y})= & \frac{a x_{m+1} y_{m+1}}{\sigma(a)\left(a x_{m+1}+y_{m+1}\right)\left(a y_{m+1}+x_{m+1}\right)} \times \\
& \times\left[Z(m+1 ; \boldsymbol{x}, \boldsymbol{y}) Z_{\mathrm{HT}}^{(2)}\left(2 m ; \boldsymbol{x} \backslash x_{m+1}, \boldsymbol{y} \backslash y_{m+1}\right)+\right. \\
& \left.+Z\left(m ; \boldsymbol{x} \backslash x_{m+1}, \boldsymbol{y} \backslash y_{m+1}\right) Z_{\mathrm{HT}}^{(2)}(2 m+2 ; \boldsymbol{x}, \boldsymbol{y})\right] .
\end{aligned}
$$

ДокАЗАТЕЛЬСтво. Сначала рассмотрим модифицированную статистическую сумму $\widetilde{Z}_{\mathrm{HT}}(2 m+1 ; \boldsymbol{x}, \boldsymbol{y})$. Сравнение рекуррентных соотношений (15) и (19) с рекуррентными соотношениями (2) и (10) наводит на мысль использовать для нее следующий анзац:

$$
\begin{aligned}
\widetilde{Z}_{\mathrm{HT}}(2 m+1 ; \boldsymbol{x}, \boldsymbol{y})= & A\left(x_{m+1}, y_{m+1}\right) \widetilde{Z}(m+1 ; \boldsymbol{x}, \boldsymbol{y}) \widetilde{Z}_{\mathrm{HT}}^{(2)}\left(2 m ; \boldsymbol{x} \backslash x_{m+1}, \boldsymbol{y} \backslash y_{m+1}\right)+ \\
& +B\left(x_{m+1}, y_{m+1}\right) \widetilde{Z}\left(m ; \boldsymbol{x} \backslash x_{m+1}, \boldsymbol{y} \backslash y_{m+1}\right) \widetilde{Z}_{\mathrm{HT}}^{(2)}(2 m+2 ; \boldsymbol{x}, \boldsymbol{y}) .
\end{aligned}
$$

Этот анзац удовлетворяет рекуррентным соотношениям (15) при условии, что функциональная форма коэффициентов $A\left(x_{m+1}, y_{m+1}\right)$ и $B\left(x_{m+1}, y_{m+1}\right)$ одинакова для всех $m$. Сравнивая члены максимальной общей степени по всем спектральным параметрам, кроме $x_{m+1}$ и $y_{m+1}$, в обеих сторонах вышеприведенного равенства и используя соотношения (20), (5), (13), (4) и (12), приходим к условию

$$
\begin{aligned}
& \frac{1}{\sigma(a)}\left[\sigma\left(a^{2 m+1}\right) x_{m+1}^{2}-\sigma\left(a^{2 m}\right) x_{m+1} y_{m+1}\right]= \\
& \quad=A\left(x_{m+1}, y_{m+1}\right)\left[\sigma\left(a^{2 m+2}\right) x_{m+1}^{2}-\sigma\left(a^{2 m}\right) y_{m+1}^{2}\right]+ \\
& \quad+B\left(x_{m+1}, y_{m+1}\right) x_{m+1}^{2}\left[\sigma\left(a^{2 m+1}\right) x_{m+1}^{2}-\sigma\left(a^{2 m-1}\right) y_{m+1}^{2}\right] .
\end{aligned}
$$

Можно убедиться, что это условие выполняется для каждого $m$ тогда и только тогда, когда

$$
\begin{aligned}
& A\left(x_{m}, y_{m}\right)=\frac{a x_{m+1} y_{m+1}}{\sigma(a)\left(a x_{m+1}+y_{m+1}\right)\left(a y_{m+1}+x_{m+1}\right)}, \\
& B\left(x_{m}, y_{m}\right)=\frac{a}{\sigma(a)\left(a x_{m+1}+y_{m+1}\right)\left(a y_{m+1}+x_{m+1}\right)} .
\end{aligned}
$$

Принимая во внимание лемму 17, заключаем, что мы получили правильное выражение для модифицированной статистической суммы $\widetilde{Z}_{\mathrm{HT}}(2 m+1 ; \boldsymbol{x}, \boldsymbol{y})$. Переходя к функциям без тильд, видим, что соотношение (21) справедливо.

3.6. Два типа матриц чередующихся знаков, симметричных относительно поворота на $180^{\circ}$. Как мы упоминали выше, матрица чередующихся знаков нечетного порядка, симметричная относительно поворота на $180^{\circ}$, имеет в центре 1 или -1 . Оказывается, что можно разделить вклады этих двух типов матриц в 
статистическую сумму. Для этого заметим, что веса вершин, соответствующих матричным элементам, равным 1 или -1 , являются четными функциями параметра $a$, в то время как веса вершин, соответствующих матричным элементам, равным 0 , являются нечетными функциями параметра $a$. Пусть число матричных элементов, равных -1 , в матрице порядка $n \times n$ равно $k$, тогда число матричных элементов, равных 1 , в этой матрице равно $n+k$. Следовательно, число матричных элементов, равных 0 , есть $n(n-1)-2 k$. Это число всегда четно, и мы имеем

$$
\left.Z(m ; \boldsymbol{x}, \boldsymbol{y})\right|_{a \rightarrow-a}=Z(m ; \boldsymbol{x}, \boldsymbol{y}) .
$$

Аналогичное рассмотрение случая матриц чередующихся знаков четного порядка, симметричных относительно поворота на $180^{\circ}$, приводит нас к заключению, что

$$
\left.Z_{\mathrm{HT}}(2 m ; \boldsymbol{x}, \boldsymbol{y})\right|_{a \rightarrow-a}=(-1)^{m} Z_{\mathrm{HT}}(m ; \boldsymbol{x}, \boldsymbol{y}) .
$$

Это равенство влечет, в частности, что

$$
\left.Z_{\mathrm{HT}}^{(2)}(2 m ; \boldsymbol{x}, \boldsymbol{y})\right|_{a \rightarrow-a}=(-1)^{m} Z_{\mathrm{HT}}^{(2)}(2 m ; \boldsymbol{x}, \boldsymbol{y}) .
$$

Рассмотрим теперь матрицу чередующихся знаков нечетного порядка $(2 m+1) \times$ $(2 m+1)$, симметричную относительно поворота на $180^{\circ}$. Пусть центральный матричный элемент этой матрицы равен 1. В этом случае число матричных элементов, равных -1 , четно. Пусть оно равно $2 l$. Тогда число матричных элементов, равных 0 , есть $(2 m+1)^{2}-(2 m+1)-4 l=2 m(2 m+1)-4 l$. Лишь половина этих нулей соответствует вершинам графа, описывающего состояние квадратного льда с границей, симметричной относительно поворота на $180^{\circ}$. Следовательно, вес рассматриваемого состояния приобретает множитель $(-1)^{m}$ при замене $a \rightarrow-a$. Аналогично мы можем доказать, что вес состояния, соответствующего матрице чередующихся знаков нечетного порядка, имеющей центральный матричный элемент, равный -1 , при этой же замене приобретает множитель $(-1)^{m+1}$. Таким образом, представляя $Z_{\mathrm{HT}}(2 m+1 ; \boldsymbol{x}, \boldsymbol{y})$ в виде

$$
Z_{\mathrm{HT}}(2 m+1 ; \boldsymbol{x}, \boldsymbol{y})=Z_{\mathrm{HT}}^{(+)}(2 m+1 ; \boldsymbol{x}, \boldsymbol{y})+Z_{\mathrm{HT}}^{(-)}(2 m+1 ; \boldsymbol{x}, \boldsymbol{y}),
$$

где

$$
\begin{aligned}
& \left.Z_{\mathrm{HT}}^{(+)}(2 m+1 ; \boldsymbol{x}, \boldsymbol{y})\right|_{a \rightarrow-a}=(-1)^{m} Z_{\mathrm{HT}}^{(+)}(2 m+1 ; \boldsymbol{x}, \boldsymbol{y}), \\
& \left.Z_{\mathrm{HT}}^{(-)}(2 m+1 ; \boldsymbol{x}, \boldsymbol{y})\right|_{a \rightarrow-a}=(-1)^{m+1} Z_{\mathrm{HT}}^{(-)}(2 m+1 ; \boldsymbol{x}, \boldsymbol{y}),
\end{aligned}
$$

мы разделяем вклады двух типов матриц. Ясно, что

$$
\begin{aligned}
& Z_{\mathrm{HT}}^{(+)}(2 m+1 ; \boldsymbol{x}, \boldsymbol{y})=\frac{1}{2}\left[Z_{\mathrm{HT}}(2 m+1 ; \boldsymbol{x}, \boldsymbol{y})+\left.(-1)^{m} Z_{\mathrm{HT}}(2 m+1 ; \boldsymbol{x}, \boldsymbol{y})\right|_{a \rightarrow-a}\right], \\
& Z_{\mathrm{HT}}^{(-)}(2 m+1 ; \boldsymbol{x}, \boldsymbol{y})=\frac{1}{2}\left[Z_{\mathrm{HT}}(2 m+1 ; \boldsymbol{x}, \boldsymbol{y})-\left.(-1)^{m} Z_{\mathrm{HT}}(2 m+1 ; \boldsymbol{x}, \boldsymbol{y})\right|_{a \rightarrow-a}\right] .
\end{aligned}
$$

Используя соотношение (21) и принимая во внимание равенства (22) и (23), мы приходим к следующей теореме. 
ТЕОрема 2. Вкладъ в статистическую суммму $Z_{\mathrm{HT}}(2 m+1 ; \boldsymbol{x}, \boldsymbol{y})$ от состояний, соответствующих матрицам, имеющим в чентре 1 или -1, равны

$$
\begin{aligned}
& Z_{\mathrm{HT}}^{(+)}(2 m+1 ; \boldsymbol{x}, \boldsymbol{y})=\frac{1}{\sigma(a) \sigma\left(a x_{m+1} \bar{y}_{m+1}\right) \sigma\left(a \bar{x}_{m+1} y_{m+1}\right)} \times \\
& \quad \times\left[(a+\bar{a}) Z(m+1 ; \boldsymbol{x}, \boldsymbol{y}) Z_{\mathrm{HT}}^{(2)}\left(2 m ; \boldsymbol{x} \backslash x_{m+1}, \boldsymbol{y} \backslash y_{m+1}\right)-\right. \\
&\left.-\left(x_{m+1} \bar{y}_{m+1}+\bar{x}_{m+1} y_{m+1}\right) Z\left(m ; \boldsymbol{x} \backslash x_{m+1}, \boldsymbol{y} \backslash y_{m+1}\right) Z_{\mathrm{HT}}^{(2)}(2 m+2 ; \boldsymbol{x}, \boldsymbol{y})\right], \quad(24) \\
& Z_{\mathrm{HT}}^{(-)}(2 m+1 ; \boldsymbol{x}, \boldsymbol{y})=\frac{1}{\sigma(a) \sigma\left(a x_{m+1} \bar{y}_{m+1}\right) \sigma\left(a \bar{x}_{m+1} y_{m+1}\right)} \times \\
& \times\left[-\left(x_{m+1} \bar{y}_{m+1}+\bar{x}_{m+1} y_{m+1}\right) Z(m+1 ; \boldsymbol{x}, \boldsymbol{y}) Z_{\mathrm{HT}}^{(2)}\left(2 m ; \boldsymbol{x} \backslash x_{m+1}, \boldsymbol{y} \backslash y_{m+1}\right)+\right. \\
&\left.\quad+(a+\bar{a}) Z\left(m ; \boldsymbol{x} \backslash x_{m+1}, \boldsymbol{y} \backslash y_{m+1}\right) Z_{\mathrm{HT}}^{(2)}(2 m+1 ; \boldsymbol{x}, \boldsymbol{y})\right],
\end{aligned}
$$

соответственно.

\section{4. СПЕЦИАЛЬНЫЕ ДЕТЕРМИНАНТНЫЕ ПРЕДСТАВЛЕНИЯ}

Оказывается, что в случае $a=e^{i \pi / 3}$ статистические суммы многих моделей квадратного льда имеют добавочные симметрийные свойства и новые представления. Интересно рассмотреть с этой точки зрения модель квадратного льда с границей, симметричной относительно поворота на $180^{\circ}$. Примем, что $a=e^{i \pi / 3}$, и обсудим сначала добавочные симметрийные свойства статистических сумм $Z(n ; \boldsymbol{x}, \boldsymbol{y})$ и $Z_{\mathrm{HT}}^{(2)}(2 m ; \boldsymbol{x}, \boldsymbol{y})$, возникающие в этом случае.

Рассмотрим статистическую сумму $Z(n ; \boldsymbol{x}, \boldsymbol{y})$ как функцию $2 n$-мерного вектора $\boldsymbol{u}=\left(u_{1}, \ldots, u_{2 n}\right)$, где $u_{2 i-1}=x_{i}$ и $u_{2 i}=y_{i}$. Из рекуррентных соотношений (3) получаем, что если $u_{2 n}=a u_{2 n-1}$, то

$$
Z(n ; \boldsymbol{u})=\sigma(a) \prod_{\mu=1}^{2 n-2} \sigma\left(a u_{\mu} \bar{u}_{2 n-1}\right) Z\left(n-1 ; \boldsymbol{u} \backslash u_{2 n-1} \backslash u_{2 n}\right)
$$

Можно показать также, что для любого $\mu=1, \ldots, 2 n$ статистическая сумма $Z(n ; \boldsymbol{u})$ удовлетворяет соотношению

$$
\begin{gathered}
Z\left(n ;\left(u_{1}, \ldots, u_{\mu}, \ldots, u_{2 n}\right)\right) \prod_{\nu \neq \mu} \sigma\left(u_{\nu} \bar{u}_{\mu}\right)+Z\left(n ;\left(u_{1}, \ldots, a^{2} u_{\mu}, \ldots, u_{2 n}\right)\right) \prod_{\nu \neq \mu} \sigma\left(u_{\nu} \bar{a}^{2} \bar{u}_{\mu}\right)+ \\
+Z\left(n ;\left(u_{1}, \ldots, \bar{a}^{2} u_{\mu}, \ldots, u_{2 n}\right)\right) \prod_{\nu \neq \mu} \sigma\left(u_{\nu} a^{2} \bar{u}_{\mu}\right)=0 .
\end{gathered}
$$

Эти соотношения позволяют получить следующее детерминантное представление для статистической суммы $Z(n ; \boldsymbol{u})$ :

$$
Z(n ; \boldsymbol{u})=(-1)^{n(n-1) / 2} \frac{\sigma^{n}(a)}{\prod_{\mu<\nu} \sigma\left(u_{\mu} \bar{u}_{\nu}\right)} \operatorname{det} P(n ; \boldsymbol{u}),
$$


где

$$
P(n ; \boldsymbol{u})=\left(\begin{array}{ccccccc}
u_{1}^{3 n-2} & u_{2}^{3 n-2} & u_{3}^{3 n-2} & u_{4}^{3 n-2} & \ldots & u_{2 n-1}^{3 n-2} & u_{2 n}^{3 n-2} \\
u_{1}^{3 n-4} & u_{2}^{3 n-4} & u_{3}^{3 n-4} & u_{4}^{3 n-4} & \ldots & u_{2 n-1}^{3 n-4} & u_{2 n}^{3 n-4} \\
u_{1}^{3 n-8} & u_{2}^{3 n-8} & u_{3}^{3 n-8} & u_{4}^{3 n-8} & \ldots & u_{2 n-1}^{3 n-8} & u_{2 n}^{3 n-8} \\
u_{1}^{3 n-10} & u_{2}^{3 n-10} & u_{3}^{3 n-10} & u_{4}^{3 n-10} & \ldots & u_{2 n-1}^{3 n-10} & u_{2 n}^{3 n-10} \\
\vdots & \vdots & \vdots & \vdots & \ddots & \vdots & \vdots \\
u_{1}^{-3 n+4} & u_{2}^{-3 n+4} & u_{3}^{-3 n+4} & u_{4}^{-3 n+4} & \ldots & u_{2 n-1}^{-3 n+4} & u_{2 n}^{-3 n+4} \\
u_{1}^{-3 n+2} & u_{2}^{-3 n+2} & u_{3}^{-3 n+2} & u_{4}^{-3 n+2} & \ldots & u_{2 n-1}^{-3 n+2} & u_{2 n}^{-3 n+2}
\end{array}\right)
$$

см. [7]. Из этого представления следует, что статистическая сумма $Z(n ; \boldsymbol{u})$ симметрична по координатам вектора $\boldsymbol{u}$.

Для функции $Z_{\mathrm{HT}}^{(2)}(2 m ; \boldsymbol{x}, \boldsymbol{y})$, рассматриваемой как функция $2 m$-мерного вектора $\boldsymbol{u}=\left(u_{1}, \ldots, u_{2 m}\right)$, при $a=e^{i \pi / 3}$ получаются такие же соотношения

$$
Z_{\mathrm{HT}}^{(2)}(2 m ; \boldsymbol{u})=\sigma(a) \prod_{\mu=1}^{2 m-2} \sigma\left(a u_{\mu} \bar{u}_{2 m-1}\right) Z_{\mathrm{HT}}^{(2)}\left(2 m-2 ; \boldsymbol{u} \backslash u_{2 m-1} \backslash u_{2 m}\right)
$$

и

$$
\begin{aligned}
Z_{\mathrm{HT}}^{(2)}(2 m ; & \left.\left(u_{1}, \ldots, u_{\mu}, \ldots, u_{2 m}\right)\right) \prod_{\nu \neq \mu} \sigma\left(u_{\nu} \bar{u}_{\mu}\right)+ \\
& +Z_{\mathrm{HT}}^{(2)}\left(2 m ;\left(u_{1}, \ldots, a^{2} u_{\mu}, \ldots, u_{2 m}\right)\right) \prod_{\nu \neq \mu} \sigma\left(u_{\nu} \bar{a}^{2} \bar{u}_{\mu}\right)+ \\
& +Z_{\mathrm{HT}}^{(2)}\left(2 m ;\left(u_{1}, \ldots, \bar{a}^{2} u_{\mu}, \ldots, u_{2 m}\right)\right) \prod_{\nu \neq \mu} \sigma\left(u_{\nu} a^{2} \bar{u}_{\mu}\right)=0
\end{aligned}
$$

как и для статистической суммы $Z(n ; \boldsymbol{u})$. Они приводят к детерминантному представлению [8]

$$
Z_{\mathrm{HT}}^{(2)}(2 m ; \boldsymbol{u})=(-1)^{m(m-1) / 2} \frac{\sigma^{m}(a)}{\prod_{\mu<\nu} \sigma\left(u_{\mu} \bar{u}_{\nu}\right)} \operatorname{det} Q(m ; \boldsymbol{u}),
$$

где

$$
Q(m ; \boldsymbol{u})=\left(\begin{array}{ccccccc}
u_{1}^{3 m-1} & u_{2}^{3 m-1} & u_{3}^{3 m-1} & u_{4}^{3 m-1} & \ldots & u_{2 m-1}^{3 m-1} & u_{2 m}^{3 m-1} \\
u_{1}^{3 m-5} & u_{2}^{3 m-5} & u_{3}^{3 m-5} & u_{4}^{3 m-5} & \ldots & u_{2 m-1}^{3 m-5} & u_{2 m}^{3 m-5} \\
u_{1}^{3 m-7} & u_{2}^{3 m-7} & u_{3}^{3 m-7} & u_{4}^{3 m-7} & \ldots & u_{2 m-1}^{3 m-7} & u_{2 m}^{3 m-7} \\
u_{1}^{3 m-11} & u_{2}^{3 m-11} & u_{3}^{3 m-11} & u_{4}^{3 m-11} & \ldots & u_{2 m-1}^{3 m-11} & u_{2 m}^{3 m-11} \\
\vdots & \vdots & \vdots & \vdots & \ddots & \vdots & \vdots \\
u_{1}^{-3 m+5} & u_{2}^{-3 m+5} & u_{3}^{-3 m+5} & u_{4}^{-3 m+5} & \ldots & u_{2 m-1}^{-3 m+5} & u_{2 m}^{-3 m+5} \\
u_{1}^{-3 m+1} & u_{2}^{-3 m+1} & u_{3}^{-3 m+1} & u_{4}^{-3 m+1} & \ldots & u_{2 m-1}^{-3 m+1} & u_{2 m}^{-3 m+1}
\end{array}\right) .
$$

Мы вновь получаем симметрию по координатам вектора $\boldsymbol{u}$. Следовательно, статистическая сумма $Z_{\mathrm{HT}}(2 m ; \boldsymbol{x}, \boldsymbol{y})$ при $a=e^{i \pi / 3}$ симметрична по объединению координат векторов $\boldsymbol{x}$ и $\boldsymbol{y}$. 
С другой стороны, статистическая сумма $Z_{\mathrm{HT}}(2 m+1 ; \boldsymbol{x}, \boldsymbol{y})$ при $a=e^{i \pi / 3}$ не является симметричной функцией векторов $\boldsymbol{x}$ и $\boldsymbol{y}$. Однако мы можем приравнять координаты $x_{m+1}=y_{m+1}$ и рассмотреть функцию

$$
Z_{\mathrm{HT}}^{\prime}(2 m+1 ; \boldsymbol{u})=Z_{\mathrm{HT}}\left(2 m+1 ;\left(u_{1}, u_{3}, \ldots, u_{2 m-1}, u_{2 m+1}\right),\left(u_{2}, u_{4}, \ldots, u_{2 m}, u_{2 m+1}\right)\right),
$$

где $\boldsymbol{u}=\left(u_{1}, \ldots, u_{2 m+1}\right)$. Как утверждает следующая теорема, функция $Z_{\mathrm{HT}}^{\prime}(2 m+1 ; \boldsymbol{u})$ симметрична по координатам вектора $\boldsymbol{u}$.

ТЕОРЕма 3. Функиия $Z_{\mathrm{HT}}^{\prime}(2 m+1 ; \boldsymbol{u})$ имеет следующее детерминантное представление:

$$
Z_{\mathrm{HT}}^{\prime}(2 m+1 ; \boldsymbol{u})=\frac{\sigma^{2 m}(a)}{\prod_{\mu<\nu} \sigma^{2}\left(u_{\mu} \bar{u}_{\nu}\right)} \operatorname{det} P^{\prime}(m+1 ; \boldsymbol{u}) \operatorname{det} P^{\prime}(m+1 ; \overline{\boldsymbol{u}}),
$$

где $P^{\prime}(m ; \boldsymbol{u})$ является $((2 m-1) \times(2 m-1))$-матрицей, которая получается из $P(m ; \boldsymbol{u})$, если удалить из нее последний столбеи, и последнюю строку.

ДокАЗАТЕЛЬСтво. Из равенства (21) следует, что

$$
\begin{aligned}
Z_{\mathrm{HT}}^{\prime}(2 m+ & 1 ; \boldsymbol{u})=\frac{1}{\sigma^{3}(a)} \times \\
& \times\left[Z\left(m+1 ;\left(u_{1}, \ldots, u_{2 m}, u_{2 m+1}, u_{2 m+1}\right)\right) Z_{\mathrm{HT}}^{(2)}\left(2 m ;\left(u_{1}, \ldots, u_{2 m}\right)\right)+\right. \\
& \left.+Z_{\mathrm{HT}}^{(2)}\left(2 m+2 ;\left(u_{1}, \ldots, u_{2 m}, u_{2 m+1}, u_{2 m+1}\right)\right) Z\left(m ;\left(u_{1}, \ldots, u_{2 m}\right)\right)\right] .
\end{aligned}
$$

Умножим уравнение (27) при $n=m$ и $\mu=2 m$ на $Z_{\mathrm{HT}}\left(m ;\left(u_{1}, \ldots, \bar{a}^{2} u_{2 m}\right)\right)$, уравнение $(30)$ при $\mu=2 m$ - на $Z\left(m ;\left(u_{1}, \ldots, \bar{a}^{2} u_{2 m}\right)\right)$ и найдем разность полученных выражений. Результат может быть записан как

$$
\frac{W\left(m ;\left(u_{1}, \ldots, u_{2 m-1}, u_{2 m}\right)\right)}{\prod_{\nu=1}^{2 m-1} \sigma\left(u_{\nu} \bar{u}_{2 m}\right)}=\frac{W\left(m ;\left(u_{1}, \ldots, u_{2 m-1}, a^{2} u_{2 m}\right)\right)}{\prod_{\nu=1}^{2 m-1} \sigma\left(u_{\nu} \bar{a}^{2} \bar{u}_{2 m}\right)},
$$

где мы ввели "вронскиан"

$$
\begin{aligned}
W(m ; \boldsymbol{u})=Z & \left(m ;\left(u_{1}, \ldots, u_{2 m-1}, \bar{a}^{2} u_{2 m}\right)\right) Z_{\mathrm{HT}}^{(2)}\left(2 m ;\left(u_{1}, \ldots, u_{2 m-1}, a^{2} u_{2 m}\right)\right)- \\
& -Z_{\mathrm{HT}}^{(2)}\left(2 m ;\left(u_{1}, \ldots, u_{2 m-1}, \bar{a}^{2} u_{2 m}\right)\right) Z\left(m ;\left(u_{1}, \ldots, u_{2 m-1}, a^{2} u_{2 m}\right)\right) .
\end{aligned}
$$

Функция $W(m ; \boldsymbol{u})$ является центрированным полиномом Лорана по $u_{2 m}$ ширины $2 m-1$. Произведение $\prod_{\mu=1}^{2 m-1} \sigma\left(u_{\mu} \bar{u}_{2 m}\right)$ является центрированным полиномом Лорана той же ширины. Умножая числители и знаменатели дробей в обеих сторонах равенства (34) на $u_{2 m}^{2 m-1}$, мы получаем, что эти дроби являются рациональными функциями переменной $u_{2 m}^{2}$. Положения возможных полюсов по $u_{2 m}^{2}$ в левой и в правой сторонах равенства (34) различны. Это означает, что равенство может выполняться лишь при условии, что рассматриваемые рациональные функции не зависят от $u_{2 m}^{2}$. Имеем, таким образом,

$$
W(m ; \boldsymbol{u})=w\left(m ; \boldsymbol{u} \backslash u_{2 m}\right) \prod_{\mu=1}^{2 m-1} \sigma\left(u_{\mu} \bar{u}_{2 m}\right) .
$$


Используя равенства

$$
\begin{aligned}
Z\left(m ;\left(u_{1}, \ldots,-u_{\mu}, \ldots, u_{2 m}\right)\right) & =(-1)^{m-1} Z\left(m ;\left(u_{1}, \ldots, u_{\mu}, \ldots, u_{2 m}\right)\right), \\
Z_{\mathrm{HT}}^{(2)}\left(m ;\left(u_{1}, \ldots,-u_{\mu}, \ldots, u_{2 m}\right)\right) & =(-1)^{m} Z_{\mathrm{HT}}^{(2)}\left(m ;\left(u_{1}, \ldots, u_{\mu}, \ldots, u_{2 m}\right)\right)
\end{aligned}
$$

и рекуррентные соотношения (26) и (29), мы можем убедиться в том, что

$$
\begin{aligned}
W\left(m ;\left(u_{1}, \ldots, u_{2 m-2}, u_{2 m-1}, a^{2} u_{2 m-1}\right)\right)=(-1)^{m} \sigma(a) \prod_{\mu=1}^{2 m-2} \sigma\left(a u_{\mu} \bar{u}_{2 m-1}\right) \times \\
\times\left[Z\left(m ;\left(u_{1}, \ldots, u_{2 m_{2}}, u_{2 m-1}, u_{2 m-1}\right)\right) Z_{\mathrm{HT}}^{(2)}\left(2 m-2 ;\left(u_{1}, \ldots, u_{2 m-2}\right)\right)+\right. \\
\left.+Z_{\mathrm{HT}}^{(2)}\left(2 m ;\left(u_{1}, \ldots, u_{2 m-2}, u_{2 m-1}, u_{2 m-1}\right)\right) Z\left(m-1 ;\left(u_{1}, \ldots, u_{2 m-2}\right)\right)\right] .
\end{aligned}
$$

С другой стороны, из соотношения (35) следует, что

$$
W\left(m ;\left(u_{1}, \ldots, u_{2 m-1}, a^{2} u_{2 m-1}\right)\right)=-\sigma(a) \prod_{\mu=1}^{2 m-2} \sigma\left(a u_{\mu} \bar{u}_{2 m-1}\right) w\left(m,\left(u_{1}, \ldots, u_{2 m-1}\right)\right) .
$$

Последние два равенства вместе с (33) дают

$$
Z_{\mathrm{HT}}(2 m+1 ; \boldsymbol{u})=\frac{(-1)^{m+1}}{\sigma^{3}(a)} w(m+1 ; \boldsymbol{u})
$$

Из детерминантных представлений (28) и (31) можно получить, что при $u_{2 m} \rightarrow 0$

$$
\begin{aligned}
Z(m ; \boldsymbol{u}) & \sim \frac{1}{u_{2 m}^{m-1}} \frac{(-1)^{m(m-1) / 2} \sigma^{m}(a)}{\prod_{\mu=1}^{2 m-1} u_{\mu} \prod_{\substack{\mu, \nu=1 \\
\mu<\nu}}^{2 m-1} \sigma\left(u_{\mu} \bar{u}_{\nu}\right)} \operatorname{det} P^{\prime}\left(m ; \boldsymbol{u} \backslash u_{2 m}\right)+\cdots, \\
Z_{\mathrm{HT}}^{(2)}(2 m ; \boldsymbol{u}) & \sim \frac{1}{u_{2 m}^{m}} \frac{(-1)^{m(m-1) / 2} \sigma^{m}(a)}{\prod_{\mu=1}^{2 m-1} u_{\mu} \prod_{\substack{\mu, \nu=1 \\
\mu<\nu}}^{2 m-1} \sigma\left(u_{\mu} \bar{u}_{\nu}\right)} \operatorname{det} Q^{\prime}\left(m ; \boldsymbol{u} \backslash u_{2 m}\right)+\cdots,
\end{aligned}
$$

где $Q^{\prime}(m ; \boldsymbol{u})$ является $((2 m-1) \times(2 m-1))$-матрицей, которая получается из $Q(m ; \boldsymbol{u})$ при удалении последнего столбца и последней строки. Эти соотношения показывают, что

$$
W(m ; \boldsymbol{u}) \sim-\frac{1}{u_{2 m}^{2 m-1}} \frac{\sigma^{2 m+1}(a)}{\prod_{\mu=1}^{2 m-1} u_{\mu}^{2} \prod_{\substack{\mu, \nu=1 \\ \mu<\nu}}^{2 m-1} \sigma^{2}\left(u_{\mu} \bar{u}_{\nu}\right)} \operatorname{det} P^{\prime}\left(m ; \boldsymbol{u} \backslash u_{2 m}\right) \operatorname{det} Q^{\prime}\left(m ; \boldsymbol{u} \backslash u_{2 m}\right)+\cdots
$$

при $u_{2 m} \rightarrow 0$. Используя равенство

$$
\operatorname{det} P^{\prime}(m ; \overline{\boldsymbol{u}})=(-1)^{m} \prod_{\mu=1}^{2 m-1} u_{\mu}^{-3} \operatorname{det} Q^{\prime}(m ; \boldsymbol{u})
$$


и соотношение (36), мы получаем

$$
w(m ; \boldsymbol{u})=(-1)^{m} \frac{\sigma^{2 m+1}(a)}{\prod_{\mu<\nu} \sigma^{2}\left(u_{\mu} \bar{u}_{\nu}\right)} \operatorname{det} P^{\prime}(m ; \boldsymbol{u}) \operatorname{det} P^{\prime}(m ; \overline{\boldsymbol{u}}) .
$$

Утверждение теоремы следует теперь из (37).

Используя совершенно другой подход, Окада также получил детерминантные представления (28) и (31) [9]. Он выразил результаты в терминах характеров классических групп и предположил, в частности, что число матриц чередующихся знаков нечетного порядка, симметричных относительно поворота на $180^{\circ}$, связано с размерностью некоторого специального представления $\mathrm{GL}(2 n+1) \times \mathrm{GL}(2 n+1)$. Возможные обобщения этой гипотезы обсуждались Купербергом [10]. Мы надеемся, что наше детерминантное представление (32) прольет дополнительный свет на этот вопрос.

\section{5. ПЕРЕЧИСЛЕНИЯ МАТРИЦ}

5.1. Детальное $x$-перечисление. Обозначим через $A(n ; x)$ полный вес $(n \times n)$ матриц чередующихся знаков, где вес индивидуальной матрицы принимается равным $x^{k}$, если она имеет $k$ матричных элементов, равных -1 . Величины $A(n ; x)$ называются $x$-перечислением матриц чередующихся знаков ${ }^{4)}$. Рассматривая статистическую сумму $Z(n ; \boldsymbol{x}, \boldsymbol{y})$ при $\boldsymbol{x}=\boldsymbol{y}=\mathbf{1}$, где $\mathbf{1}=(1, \ldots, 1)$, мы получаем информацию о $x$-перечислении. А именно, справедливо равенство

$$
A(n ; x)=\frac{1}{\sigma^{n^{2}-n}(a) \sigma^{n}\left(a^{2}\right)} Z(n ; \mathbf{1}, \mathbf{1}),
$$

где

$$
x=\left[\frac{\sigma\left(a^{2}\right)}{\sigma(a)}\right]^{2}=(a+\bar{a})^{2} .
$$

В частности, если $a=e^{i \pi / 3}$, то $x=1$, и приведенное равенство дает общее число $(n \times n)$-матриц чередующихся знаков $A(n)=A(n, 1)$. В статье [2] Куперберг использовал детерминантное представление Изергина-Корепина для статистической суммы $Z(n ; \boldsymbol{x}, \boldsymbol{y})[11]$ и привел новое элегантное доказательство формулы для $A(n)$, предложенной Миллсом, Роббинсом и Рамсеем [1] и впервые доказанной Цайлбергером [12].

Можно также определить детальное $x$-перечисление матриц чередующихся знаков. Напомним, что матрица чередующихся знаков имеет только одну 1 в первом столбце, а все остальные элементы этого столбца равны нулю. Обозначим через $A(n, r ; x)$ полный вес $(n \times n)$-матриц чередующихся знаков, имеющих 1 в $r$-й позиции первого столбца. При этом вес отдельной матрицы принимается равным $x^{k}$, если она имеет $k$ матричных элементов, равных -1 . Легко видеть, что

$$
\mathcal{A}(n ; t, x) \equiv \sum_{r=1}^{n} A(n, r ; x) t^{r-1}=\frac{Z(n ; \mathbf{1},(v, 1, \ldots, 1))}{\sigma^{n^{2}-2 n+1}(a) \sigma^{n}\left(a^{2}\right) \sigma^{n-1}(a v)},
$$

\footnotetext{
4) Не следует путать вектор $\boldsymbol{x}$ и параметр перечисления $x$.
} 
где

$$
x=\left[\frac{\sigma\left(a^{2}\right)}{\sigma(a)}\right]^{2}, \quad t=\frac{\sigma(a \bar{v})}{\sigma(a v)} .
$$

Если $a=e^{i \pi / 3}$, то мы имеем формулы для детального перечисления матриц чередующихся знаков: $A(n, r)=A(n, r ; 1)$. В статье [13] Цайлбергер использовал детерминантное представление Изергина-Корепина для статистической суммы $Z(n ; \boldsymbol{x}, \boldsymbol{y})$, чтобы доказать гипотезу Миллса, Роббинса и Рамсея [1] для детального перечисления матриц чередующихся знаков $A(n, r)$.

Для случая матриц чередующихся знаков четного порядка, симметричных относительно поворота на $180^{\circ}$,

$$
\mathcal{A}_{\mathrm{HT}}(2 m ; t, x) \equiv \sum_{r=1}^{2 m} A_{\mathrm{HT}}(2 m, r ; x) t^{r-1}=\frac{Z_{\mathrm{HT}}(2 m ; \mathbf{1},(v, 1, \ldots, 1))}{\sigma^{2 m^{2}-3 m+1}(a) \sigma^{m}\left(a^{2}\right) \sigma^{2 m-1}(a v)} .
$$

Отметим, что здесь вес отдельной матрицы равен $x^{k / 2}$, если она имеет $k$ матричных элементов, равных -1 . Так как в рассматриваемом случае число таких элементов всегда четно, то фактически вес матрицы определяется числом орбит симметрии матричных элементов, равных -1 . Удобно ввести обозначение

$$
\mathcal{A}_{\mathrm{HT}}^{(2)}(2 m ; t, x) \equiv \frac{\mathcal{A}_{\mathrm{HT}}(2 m ; t, x)}{\mathcal{A}(m ; t, x)}=\frac{Z_{\mathrm{HT}}^{(2)}(2 m ; \mathbf{1},(v, 1, \ldots, 1))}{\sigma^{m^{2}-m}(a) \sigma^{m}(a v)} .
$$

Далее, для случая матриц чередующихся знаков нечетного порядка, симметричных относительно поворота на $180^{\circ}$, мы получаем

$$
\mathcal{A}_{\mathrm{HT}}(2 m+1 ; t, x) \equiv \sum_{r=1}^{2 m+1} A_{\mathrm{HT}}(2 m+1 ; r, x) t^{r-1}=\frac{Z_{\mathrm{HT}}(2 m+1 ; \mathbf{1},(v, 1, \ldots, 1))}{\sigma^{2 m^{2}-m}(a) \sigma^{m}\left(a^{2}\right) \sigma^{2 m}(a v)} .
$$

Здесь вновь вес отдельной матрицы равен $x^{k / 2}$, если она имеет $k$ матричных элементов, равных -1. Заметим, что число таких элементов четно или нечетно в зависимости от того, равен центральный матричный элемент 1 или -1 . В этом случае Роббинс также определял вес матрицы в соответствии с числом орбит симметрии матричных элементов, равных - 1 [4]. Наше определение представляется более удобным. Связь с $x$-перечислением, используемым Роббинсом, дается ниже.

Имея в виду представление (21), из (40), (38) и (39) мы получаем

$$
\mathcal{A}_{\mathrm{HT}}(2 m+1 ; t, x)=\frac{\sqrt{x} \mathcal{A}(m+1 ; t, x) \mathcal{A}_{\mathrm{HT}}^{(2)}(2 m ; t, x)+\mathcal{A}(m ; t, x) \mathcal{A}_{\mathrm{HT}}^{(2)}(2 m+2 ; t, x)}{\sqrt{x}+2} .
$$

В то время как $\mathcal{A}(m ; t, x)$ и $\mathcal{A}_{\mathrm{HT}}^{(2)}(m ; t, x)$ являются полиномами по переменным $x$ и $t, \mathcal{A}_{\mathrm{HT}}(2 m+1 ; t, x)$ содержит также полуцелые степени по переменной $x$. Можно разделить $\mathcal{A}_{\mathrm{HT}}(2 m+1 ; t, x)$ на две части:

$$
\mathcal{A}_{\mathrm{HT}}(2 m+1 ; t, x)=\mathcal{A}_{\mathrm{HT}}^{(+)}(2 m+1 ; t, x)+\sqrt{x} \mathcal{A}_{\mathrm{HT}}^{(-)}(2 m+1 ; t, x),
$$


где $\mathcal{A}_{\mathrm{HT}}^{(+)}(2 m+1 ; t, x)$ и $\mathcal{A}_{\mathrm{HT}}^{(-)}(2 m+1 ; t, x)$ - полиномы по переменной $x$. Полученные две части задают детальные $x$-перечисления матриц чередующихся знаков нечетного порядка, симметричных относительно поворота на $180^{\circ}$, с 1 и -1 в центре матрицы, соответственно. Соотношение (41) дает ${ }^{5)}$

$$
\begin{aligned}
\mathcal{A}_{\mathrm{HT}}^{(+)}(2 m & +1 ; t, x)= \\
& =\frac{-x \mathcal{A}(m+1 ; t, x) \mathcal{A}_{\mathrm{HT}}^{(2)}(2 m ; t, x)+2 \mathcal{A}(m ; t, x) \mathcal{A}_{\mathrm{HT}}^{(2)}(2 m+2 ; t, x)}{4-x}, \\
\mathcal{A}_{\mathrm{HT}}^{(-)}(2 m+1 ; t, x)= & \\
& =\frac{2 \mathcal{A}(m+1 ; t, x) \mathcal{A}_{\mathrm{HT}}^{(2)}(2 m ; t, x)-\mathcal{A}(m ; t, x) \mathcal{A}_{\mathrm{HT}}^{(2)}(2 m+2 ; t, x)}{4-x} .
\end{aligned}
$$

Роббинс использовал $x$-перечисление матриц чередующихся знаков, симметричных относительно поворота на $180^{\circ}$, связанное с числом орбит симметрии для матричных элементов, равных -1 [4]. Ясно, что такое $x$-перечисление имеет вид

$$
\begin{aligned}
\mathcal{A}_{\mathrm{HT}}^{\mathrm{R}}(2 m+1 ; t, x) & =\mathcal{A}_{\mathrm{HT}}^{(+)}(2 m+1 ; t, x)+x \mathcal{A}_{\mathrm{HT}}^{(-)}(2 m+1 ; t, x)= \\
& =\frac{x \mathcal{A}(m+1 ; t, x) \mathcal{A}_{\mathrm{HT}}^{(2)}(2 m ; t, x)+(2-x) \mathcal{A}(m ; t, x) \mathcal{A}_{\mathrm{HT}}^{(2)}(2 m+2 ; t, x)}{4-x} .
\end{aligned}
$$

Мы видим, что детальные $x$-перечисления $\mathcal{A}_{\mathrm{HT}}^{(+)}(2 m+1 ; t, x)$ и $\mathcal{A}_{\mathrm{HT}}^{(-)}(2 m+1 ; t, x)$ определяются полиномами $\mathcal{A}(m ; t, x)$ и $\mathcal{A}_{\mathrm{HT}}^{(2)}(2 m ; t, x)$. Эти полиномы для общего $x$ все еще неизвестны. Однако для $x=1$ мы знаем точную форму этих полиномов. Это позволяет получить выражения для детальных 1-перечислений матриц чередующихся знаков нечетного порядка, симметричных относительно поворота на $180^{\circ}$. Начнем с обычных перечислений (1-перечислений).

5.2. 1-перечисления. Напомним, что полное число матриц чередующихся знаков задается формулой

$$
A(m)=\prod_{i=0}^{m-1} \frac{(3 i+1) !}{(m+i) !}
$$

а полное число матриц чередующихся знаков четного порядка, симметричных относительно поворота на $180^{\circ}$, задается формулой

$$
A_{\mathrm{HT}}(2 m)=\prod_{i=0}^{m-1} \frac{(3 i) !(3 i+2) !}{[(m+i) !]^{2}} .
$$

Из этих соотношений следует, что

$$
\begin{gathered}
\frac{A(m+1)}{A(m)}=\frac{m !(3 m+1) !}{(2 m) !(2 m+1) !}, \\
\frac{A_{\mathrm{HT}}(2 m+2)}{A_{\mathrm{HT}}(2 m)}=\frac{[m !]^{2}(3 m) !(3 m+2) !}{[(2 m) !(2 m+1) !]^{2}} .
\end{gathered}
$$

\footnotetext{
5) Конечно, можно непосредственно использовать соотношения (24) и (25).
} 
Фиксируя $x=1$ и $t=1$ и имея в виду, что $\mathcal{A}(n ; 1,1)=A(n)$ и $\mathcal{A}_{\mathrm{HT}}^{(2)}(2 m ; 1,1)=$ $A_{\mathrm{HT}}(2 m) / A(m)$, получаем из $(42)$ и $(43)$ следующие равенства:

$$
\begin{aligned}
& \frac{A_{\mathrm{HT}}^{(+)}(2 m+1)}{A_{\mathrm{HT}}(2 m)}=-\frac{1}{3} \frac{A(m+1)}{A(m)}+\frac{2}{3} \frac{A_{\mathrm{HT}}(2 m+2)}{A_{\mathrm{HT}}(2 m)} \frac{A(m)}{A(m+1)}, \\
& \frac{A_{\mathrm{HT}}^{(-)}(2 m+1)}{A_{\mathrm{HT}}(2 m)}=\frac{2}{3} \frac{A(m+1)}{A(m)}-\frac{1}{3} \frac{A_{\mathrm{HT}}(2 m+2)}{A_{\mathrm{HT}}(2 m)} \frac{A(m)}{A(m+1)} .
\end{aligned}
$$

Используя соотношения (44) и (45), можно выразить $A_{\mathrm{HT}}^{(+)}(2 m+1)$ и $A_{\mathrm{HT}}^{(-)}(2 m+1)$ через $A_{\mathrm{HT}}(2 m)$. Удобно записать ответ в следующем виде:

$$
\begin{aligned}
& A_{\mathrm{HT}}(2 m+1)=\frac{(m) !(3 m) !}{[(2 m) !]^{2}} A_{\mathrm{HT}}(2 m), \\
& A_{\mathrm{HT}}^{(+)}(2 m+1)=\frac{m+1}{2 m+1} A_{\mathrm{HT}}(2 m+1), \\
& A_{\mathrm{HT}}^{(-)}(2 m+1)=\frac{m}{2 m+1} A_{\mathrm{HT}}(2 m+1) .
\end{aligned}
$$

Первое равенство было предложено Роббинсом [4], но, насколько мы знаем, оно пока еще не было доказано.

Достаточно неожиданной оказывается простота равенства

$$
\frac{A_{\mathrm{HT}}^{(+)}(2 m+1)}{A_{\mathrm{HT}}^{(-)}(2 m+1)}=\frac{m+1}{m} .
$$

5.3. Детальные 1-перечисления. Вид полинома $\mathcal{A}(m ; t) \equiv \mathcal{A}(m ; t, 1)$ составляет суть знаменитой гипотезы о детальном распределении матриц чередующихся знаков, предложенной Миллсом, Роббинсом и Рамсеем [1] и доказанной Цайлбергером [13]:

$$
\frac{\mathcal{A}(m ; t)}{A(m)}=\frac{(2 m-1) !}{(m-1) !(3 m-2) !} \sum_{r=1}^{m} \frac{(m+r-2) !(2 m-r-1) !}{(r-1) !(m-r) !} t^{r-1}
$$

Полином $\mathcal{A}_{\mathrm{HT}}^{(2)}(m ; t) \equiv \mathcal{A}_{\mathrm{HT}}^{(2)}(m ; t, 1)$ был найден в статье [8]:

$$
\begin{aligned}
\frac{\mathcal{A}_{\mathrm{HT}}^{(2)}(2 m ; t)}{A_{\mathrm{HT}}^{(2)}(2 m)}= & \frac{(3 m-2)(2 m-1) !}{(m-1) !(3 m-1) !} \times \\
& \quad \times \sum_{r=1}^{m+1} \frac{\left(m^{2}-m r+(r-1)^{2}\right)(m+r-3) !(2 m-r-1)}{(r-1) !(m-r+1) !} t^{r-1} .
\end{aligned}
$$

Используя соотношения (42) и (43) при $x=1$, получаем детальные перечисления матриц чередующихся знаков нечетного порядка, симметричных относительно поворота на $180^{\circ}$, с 1 и -1 в центре, соответственно. 
5.4. Детальные 4-перечисления. Вернемся к соотношениям (42) и (43). Знаменатели дробей в правых частях этих соотношений равны $4-x$. С другой стороны, выражения в левых частях регулярны при любом $x$. Следовательно, должно выполняться равенство

$$
2 \mathcal{A}(m+1 ; t, 4) \mathcal{A}_{\mathrm{HT}}^{(2)}(2 m ; t, 4)=\mathcal{A}(n ; t, 4) \mathcal{A}_{\mathrm{HT}}^{(2)}(2 m+2 ; t, 4),
$$

которое влечет за собой рекуррентное соотношение

$$
2 \frac{\mathcal{A}^{2}(m+1 ; t, 4)}{\mathcal{A}^{2}(m ; t, 4)}=\frac{\mathcal{A}_{\mathrm{HT}}(2 m+2 ; t, 4)}{\mathcal{A}_{\mathrm{HT}}(2 m ; t, 4)} .
$$

Используя равенства $\mathcal{A}_{\mathrm{HT}}(2 ; t, x)=1+t$ и $\mathcal{A}(1 ; t, x)=1$, приходим к соотношению

$$
\mathcal{A}_{\mathrm{HT}}(2 m ; t, 4)=2^{m-1}(1+t) \mathcal{A}^{2}(m ; t, 4) .
$$

Благодарности. Работа была частично поддержана РФФИ (грант № 04-0100352). Мы благодарны Г. Купербергу за интересную и стимулирующую переписку.

\section{Список литературы}

[1] W. H. Mills, D. P. Robbins, H. Rumsey, Jr., Invent. Math., 66 (1982), 73-87; J. Combin. Theory Ser. A, 34 (1983), 340-359.

[2] G. Kuperberg, Int. Math. Res. Notices, 1996:3 (1996), 139-150; math.C0/9712207.

[3] G. Kuperberg, Ann. Math., 156 (2002), 835-866; math.C0/0008184.

[4] D. P. Robbins, Symmetry Classes of Alternating Sign Matrices, math.C0/0008045.

[5] V. E. Korepin, Commun. Math. Phys., 86 (1982), 391-418.

[6] D. P. Robbins, H. Rumsey, Jr., Advances in Math., 62:2 (1986), 169-184; N. Elkies, G. Kuperberg, M. Larsen, J. Propp, J. Algebraic Combin., 1 (1992), 111-132; 219-234; math.CO/9201305.

[7] Ю. Г. Строганов, ТMФ, 146:1 (2006), 65-76; math-ph/0204042; А.В. Разумов, Ю. Г. Строганов, ТМФ, 141:3 (2004), 323-347; math-ph/0312071.

[8] Yu. G. Stroganov, Izergin-Korepin determinant reloaded, math-ph/0409072.

[9] S. Okada, J. Algebraic. Combin., 23 (2006), 43-69; math.C0/0408234.

[10] G. Kuperberg, E-mail message to private "domino" forum, 10/07/2004; private communication.

[11] А. Г. Изергин, ДАН СССР, 297 (1987), 331-333; V. E. Korepin, N. M. Bogoliubov, A. G. Izergin, Quantum Inverse Scattering Method, Correlation Functions and Algebraic Bethe Ansatz, (2nd ed.), Cambridge University Press, Cambridge, 1993.

[12] D. Zeilberger, Elec. J. Comb., 3(2) (1996), R13; math.Co/9407211.

[13] D. Zeilberger, New York J. Math., 2 (1996), 59-68; math.C0/9606224. 